\title{
Analytical Solutions for the Study of Homogeneous First-Order Chemical Kinetics via UV-Vis Spectroelectrochemistry
}

\author{
A. Molina*,a, E. Laborda ${ }^{a}$, J.M. Gómez-Gila , F. Martínez-Ortiz and R.G. Compton ${ }^{\text {b }}$ \\ a Departamento de Química Física, Facultad de Química, Regional Campus of International \\ Excellence "Campus Mare Nostrum", Universidad de Murcia, 30100 Murcia, Spain \\ ${ }^{b}$ Department of Chemistry, Physical \& Theoretical Chemistry Laboratory, Oxford University, \\ South Parks Road, Oxford OX1 3QZ (UK). Fax: (+44) 1865-275-410
}

* Corresponding author:

Tel: +34868887524

Fax: +34 868884148

Email: amolina@um.es 


\section{Abstract}

Analytical solutions are reported for the identification and study of homogeneous chemical reactions via UV-vis spectroelectrochemistry. Expressions are presented for the concentration profiles and the absorbance response of any the species of the CEC mechanism when applying a potential-pulse perturbation under semi-infinite linear diffusion conditions. With this theory it is possible to relate easily the absorbance transient to the physicochemical dynamics of the redox system. In particular, the influence of the chemical reactions involving the oxidized and/or the reduced species will be investigated both in the normal and parallel beam configurations. Expressions for the reaction schemes $C E, E C$ and $E$ can be deduced as particular cases. The EC solutions are applied to the study of facilitated ion transfers across liquid | liquid interfaces where the likely different diffusivity of the ion in the two phases has been considered.

Keywords: Analytical theory; UV-vis spectroelectrochemistry; Reaction mechanism; Concentration profiles; Constant potential chronoamperometry 


\section{Introduction}

In electrochemical measurements it is frequently found that the electroactive species and their products are involved in chemical reactions in solution. Hence, for the comprehensive analysis of the charge transfer process, the reaction mechanism must be elucidated and the chemical kinetics and equilibrium constants must be determined. For this, monitoring the species involved or affected by the electrode reaction via spectroscopic methods (UV-vis, IR, Raman, EPR [1,2], etc. [3-6]) can be a very convenient, complementary approach. Indeed, the alteration of the species concentration profiles upon driving the electrode process is the ultimate response of the system. Thus, spectroelectrochemical (SEC) methods have already been successfully applied to the study of isomerisations, dissociation/association reactions and electrocatalytic processes [3], as well as to the investigation of charge transfer reactions at liquid|liquid interfaces [7].

The SEC signal is evidently a function of the concentration profiles of the absorbing species. Thus, theoretical expressions for such profiles are needed in order to derive analytical relationships between the absorbance transient and the physicochemical processes that define the dynamic response of the system (namely, mass transport, charge transfer reaction and homogeneous chemical processes in the absence of adsorption). Assuming that a beam of light is incident normal to an optically transparent macroelectrode placed in the $y z$ plane (Scheme 1a), the UV-vis absorbance response of species $i, \mathrm{~A}_{i, \mathrm{~N}}(\lambda, t)$, is proportional to the integral of its concentration profile along $x$ [8]:

$$
\mathrm{A}_{i, \mathrm{~N}}(\lambda, t)=\varepsilon_{i}(\lambda) \int_{0}^{l} c_{i}(x, t) d x
$$

where $l$ is the path length of the beam through the electrolyte and $\varepsilon_{i}(\lambda)$ is the wavelength dependent extinction coefficient of species $i$. 
(a) Normal beam configuration ( $\mathrm{N}$ )

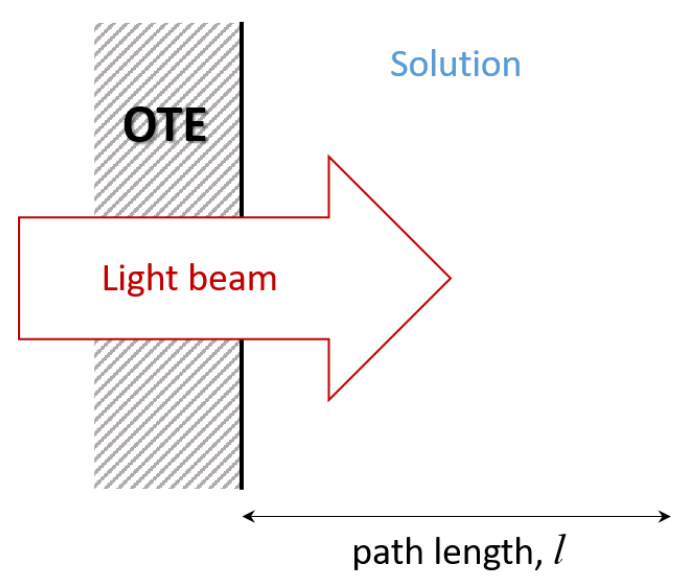

(b) Parallel beam configuration (P)

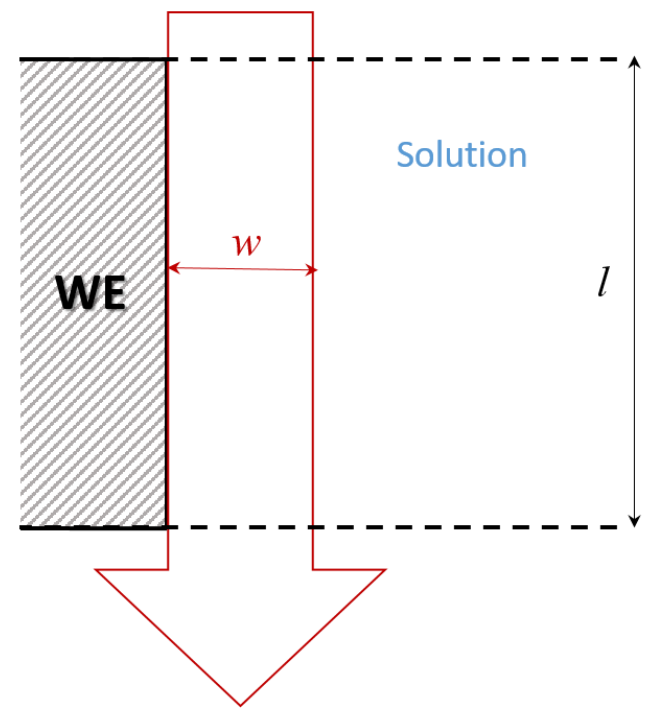

Scheme 1. Normal-beam (N) and parallel-beam (P) configurations. OTE: optically transparent electrode; WE: working electrode

When the light beam samples the solution parallel to the electrode, then [9]:

$$
\mathrm{A}_{i, \mathrm{P}}(\lambda, t)=\log \left(\frac{w}{\int_{0}^{w} 10^{-\varepsilon_{i}(\lambda) l c_{i}(x, t)} d x}\right)
$$

where $w$ is the height of the light beam in the parallel configuration and $l$ the path length over the electrode surface (Scheme 1b).

So far, analytical solutions for the SEC signal have been reported for the E and catalytic mechanisms. Thus, when the electrogenerated species $\mathrm{R}$ is the photoactive one:

$$
\mathrm{O}+e^{-} \rightleftarrows \mathrm{R}^{*}
$$

the normal beam configuration (N) absorbance of the E mechanism is given by Eq. (1) (and also by integration of the current with respect to time, that is, by the converted charge) that leads to $[8,10]:$

$$
\mathrm{A}_{\mathrm{R}, \mathrm{N}}^{\mathrm{E}}(\lambda, t)=\frac{\varepsilon_{\mathrm{R}}(\lambda)}{F A} \int_{0}^{t} I(t) d t=2 \sqrt{\frac{D t}{\pi}} \varepsilon_{i}(\lambda) c_{\mathrm{O}}^{*} \frac{1}{1+e^{\eta}}
$$


with $\eta=\frac{F}{R T}\left(E-E^{0^{\prime}}\right)$. In the parallel configuration (P), the SEC response of the $E$ mechanism under limiting current conditions and with the restrictions of large $w$-values (compared to the diffusion layer) and low absorbance [9] is given by [11]:

$$
\mathrm{A}_{\mathrm{R}, \mathrm{P}}^{\mathrm{E}, \lim }(\lambda, t)=2 \frac{l}{w} \sqrt{\frac{D t}{\pi}} \varepsilon_{\mathrm{R}}(\lambda) c_{\mathrm{O}}^{*}
$$

In the case of the first-order catalytic mechanism where the homogeneous reaction is irreversible:

$$
\begin{gathered}
\mathrm{O}+e^{-} \rightleftarrows \mathrm{R}^{*} \\
\mathrm{R}^{*}+\mathrm{Z} \stackrel{k_{c a t}}{\longrightarrow} \mathrm{O}+\mathrm{Z}^{\prime}
\end{gathered}
$$

the following expressions are obtained for normal configuration (N) SEC [12]:

$$
\mathrm{A}_{\mathrm{R}, \mathrm{N}}^{\mathrm{cat}, \lim }(\lambda, t)=\varepsilon_{\mathrm{R}}(\lambda) c_{\mathrm{O}}^{*} \sqrt{\frac{D}{k_{\mathrm{cat}} c_{Z}^{*}}} \operatorname{erf}\left(\sqrt{k_{\mathrm{cat}} c_{Z}^{*} t}\right)
$$

and for the parallel configuration with large $w$-values and low absorbance [13]:

$$
\mathrm{A}_{\mathrm{R}, \mathrm{P}}^{\mathrm{cat}, \lim }(\lambda, t)=\varepsilon_{\mathrm{R}}(\lambda) c_{\mathrm{O}}^{*} \frac{l}{w} \sqrt{\frac{D}{k_{\mathrm{cat}} c_{\mathrm{Z}}^{*}}} \operatorname{erf}\left(\sqrt{k_{\mathrm{cat}} c_{\mathrm{Z}}^{*} t}\right)
$$

For other charge transfer mechanisms, the theoretical treatment of the $\mathrm{A}(\lambda, t)$ response has been carried out by means of numerical simulations [14-17] (except for the EC mechanism under galvanostatic conditions $[18,19])$ to the best of our knowledge. Here, analytical solutions for the concentration profiles of the CEC mechanism are reported for the SEC response upon the application of a constant potential pulse:

$$
\begin{array}{cl}
Z \underset{k_{2}}{\stackrel{k_{1}}{\rightleftarrows}} O & ; K=c_{Z}^{e q} / c_{O}^{e q}=k_{2} / k_{1} \\
O+e^{-} \underset{k_{1}^{\prime}}{\rightleftarrows} R & ; E^{0^{\prime}} \\
R \underset{k_{2}^{\prime}}{\rightleftarrows} & ; K^{\prime}=c_{R}^{e q} / c_{Z^{\prime}}^{e q}=k_{2}^{\prime} / k_{1}^{\prime}
\end{array}
$$

Note that by studying the CEC mechanism we can evaluate the effects of chemical reactions affecting any of the electroactive species, as well as analyse the $\mathrm{E}\left(K \rightarrow 0, K^{\prime}>>1\right), \mathrm{CE}\left(K^{\prime}>>1\right.$ ) and $\mathrm{EC}^{\prime}(K \rightarrow 0)$ mechanisms as particular cases. As will be shown, having at our disposal 
analytical expressions for the concentration profiles will enable simple and rapid interpretation of the experimental spectro-electrochemical response. 


\section{Theoretical treatment: the CEC mechanism}

The differential equation system that describes the evolution with time of the concentration profiles of the CEC mechanism under semi-infinite, linear diffusion when applying a potential pulse $E$ is:

$$
\begin{aligned}
& \frac{\partial c_{Z}}{\partial t}=D \frac{\partial^{2} c_{Z}}{\partial x^{2}}-k_{1} c_{Z}+k_{2} c_{O} \\
& \frac{\partial c_{O}}{\partial t}=D \frac{\partial^{2} c_{O}}{\partial x^{2}}+k_{1} c_{Z}-k_{2} c_{O} \\
& \frac{\partial c_{R}}{\partial t}=D^{\prime} \frac{\partial^{2} c_{R}}{\partial x^{2}}-k_{1}^{\prime} c_{R}+k_{2}^{\prime} c_{Z^{\prime}} \\
& \frac{\partial c_{Z^{\prime}}}{\partial t}=D^{\prime} \frac{\partial^{2} c_{Z^{\prime}}}{\partial x^{2}}+k_{1}^{\prime} c_{R}-k_{2}^{\prime} c_{Z^{\prime}}
\end{aligned}
$$

$\left.\begin{array}{l}t=0, \forall x \\ t>0, x \rightarrow \infty\end{array}\right\} \begin{array}{cc}c_{\mathrm{O}}(\infty)=c_{\mathrm{O}}^{e q}, & c_{\mathrm{Z}}(\infty)=c_{\mathrm{Z}}^{e q} \\ c_{\mathrm{R}}(\infty)=0, & c_{\mathrm{Z}^{\prime}}(\infty)=0\end{array}$

$$
\begin{gathered}
D\left(\frac{\partial c_{\mathrm{O}}}{\partial x}\right)_{0}=-D^{\prime}\left(\frac{\partial c_{\mathrm{R}}}{\partial x}\right)_{0} \\
\left(\frac{\partial c_{\mathrm{Z}}}{\partial x}\right)_{0}=\left(\frac{\partial c_{\mathrm{Z}^{\prime}}}{\partial x}\right)_{0}=0
\end{gathered}
$$

where $D$ and $D^{\prime}$ are the diffusion coefficients of the oxidized ( $\mathrm{O}$ and $\mathrm{Z}$ ) and reduced ( $\mathrm{R}$ and $\mathrm{Z}^{\prime}$ ) species, respectively. Note that considering in the theory that the values of $D$ and $D^{\prime}$ can be different is important for its application to the study of ion transfer reactions between water and organic phases [7], where species may show very different diffusivity in each phase [20].

Note that it has been assumed that only the reagent species $Z$ and $O$ are initially present and also that the spectro-electrochemical cell is considerably larger than the depletion layer and semi-infinite diffusion holds (Eq. (11)). Further, the presence of sufficient supporting electrolyte to suppress migration is presumed [21] as well as that the duration of the SEC experiment is controlled to avoid the onset of natural convection [22]. 
The boundary value problem (10)-(14) will be solved analytically in this work by extending the procedure given in ref. [23], obtaining expressions for the concentration profiles of all the chemical species. First, it is convenient to define the functions:

$$
\begin{aligned}
& \varsigma=\mathrm{c}_{\mathrm{O}}+\mathrm{c}_{\mathrm{Z}} \\
& \phi=\mathrm{c}_{\mathrm{Z}}-K \mathrm{c}_{\mathrm{O}}
\end{aligned}
$$

and:

$$
\begin{aligned}
& \varsigma^{\prime}=\mathrm{c}_{\mathrm{R}}+\mathrm{c}_{\mathrm{Z}^{\prime}} \\
& \phi^{\prime}=\mathrm{c}_{\mathrm{R}}-K^{\prime} \mathrm{c}_{\mathrm{Z}^{\prime}}
\end{aligned}
$$

that fulfil the following differential equations:

$$
\begin{array}{cc}
\frac{\partial \varsigma}{\partial t}=D \frac{\partial^{2} \varsigma}{\partial x^{2}} & ; \quad \frac{\partial \varsigma^{\prime}}{\partial t}=D^{\prime} \frac{\partial^{2} \varsigma^{\prime}}{\partial x^{2}} \\
\frac{\partial \phi}{\partial t}=D \frac{\partial^{2} \phi}{\partial x^{2}}-\kappa \phi \quad ; & \frac{\partial \phi^{\prime}}{\partial t}=D^{\prime} \frac{\partial^{2} \phi^{\prime}}{\partial x^{2}}-\kappa^{\prime} \phi^{\prime}
\end{array}
$$

with:

$$
\begin{aligned}
& \kappa=k_{1}+k_{2} \\
& \kappa^{\prime}=k_{1}^{\prime}+k_{2}^{\prime}
\end{aligned}
$$

and they are subject to the following boundary conditions:

$$
\begin{aligned}
& \left.\begin{array}{l}
t=0, \forall x \\
t>0, x \rightarrow \infty
\end{array}\right\} \varsigma(\infty)=\varsigma^{*}=c_{\mathrm{O}}^{e q}+c_{\mathrm{Z}}^{e q} ; \varsigma^{\prime}(\infty)=0 ; \phi(\infty)=0 ; \phi^{\prime}(\infty)=0 \\
& t>0, x=0 \text { : } \\
& \gamma^{2}\left(\frac{\partial \varsigma(x, t)}{\partial x}\right)_{0}=-\left(\frac{\partial \varsigma^{\prime}(x, t)}{\partial x}\right)_{0} \\
& \left(\frac{\partial \varsigma(x, t)}{\partial x}\right)_{0}=-\frac{1}{K}\left(\frac{\partial \phi(x, t)}{\partial x}\right)_{0} \\
& \left(\frac{\partial \varsigma^{\prime}(x, t)}{\partial x}\right)_{0}=\left(\frac{\partial \phi^{\prime}(x, t)}{\partial x}\right)_{0} \\
& \varsigma(0, t)-\phi(0)=e^{\eta}\left(\frac{1+K}{1+K^{\prime}}\right)\left\{K^{\prime} \varsigma^{\prime}(0, t)+\phi^{\prime}(0)\right\}
\end{aligned}
$$


with

$$
\gamma^{2}=\frac{D}{D^{\prime}}
$$

In the present mathematical treatment, functions $\phi$ and $\phi^{\prime}$, which account for the magnitude of the perturbation of the chemical equilibria, are assumed to be independent of time such that Eqs. (18) become into $[23,24]$ :

$$
D \frac{\partial^{2} \phi}{\partial x^{2}}=\kappa \phi \quad ; \quad D^{\prime} \frac{\partial^{2} \phi^{\prime}}{\partial x^{2}}=\kappa^{\prime} \phi^{\prime}
$$

Then, functions $\phi$ and $\phi^{\prime}$ are given by:

$$
\phi=\phi(0) \exp \left(-\sqrt{\frac{\kappa}{D}} x\right) \quad ; \quad \phi^{\prime}=\phi^{\prime}(0) \exp \left(-\sqrt{\frac{\kappa^{\prime}}{D^{\prime}}} x\right)
$$

The above assumption has been reported to be very appropriate for different reaction mechanisms [25] since it enables us to obtain simpler expressions for the concentration profiles and the current response.

Taking into account the above, the surface boundary conditions for Eqs. (17) simplify to: $t>0, x=0$ :

$$
\begin{gathered}
\gamma^{2}\left(\frac{\partial \varsigma(x, t)}{\partial x}\right)_{0}=-\left(\frac{\partial \varsigma^{\prime}(x, t)}{\partial x}\right)_{0} \\
\left(\frac{\partial \varsigma(x, t)}{\partial x}\right)_{0}=\frac{1}{K} \frac{\phi(0)}{\delta_{r}} \\
\left(\frac{\partial \zeta^{\prime}(x, t)}{\partial x}\right)_{0}=-\frac{\phi^{\prime}(0)}{\delta_{r}^{\prime}} \\
\varsigma(0)-\phi(0)=e^{\eta}\left(\frac{1+K}{1+K^{\prime}}\right)\left\{K^{\prime} \varsigma^{\prime}(0)+\phi^{\prime}(0)\right\}
\end{gathered}
$$

where $\delta_{r}$ and $\delta_{r}^{\prime}$ are the thickness of the so-called linear reaction layers [25], which reflect the extent of the region of the solution next to the electrode|solution (or liquid|liquid) interface where chemical equilibrium conditions are perturbed by the heterogeneous charge transfer process: 


$$
\begin{aligned}
& \delta_{r}=\sqrt{\frac{D}{\kappa}} \\
& \delta_{r}^{\prime}=\sqrt{\frac{D^{\prime}}{\kappa^{\prime}}}
\end{aligned}
$$

Note that $\delta_{r}^{(1)} \leq \delta^{(1)}[25]$.

Then, substituting Eqs. (29) and (30) into Eq. (31), one obtains:

$$
\varsigma(0)-K \delta_{r}\left(\frac{\partial \varsigma}{\partial x}\right)_{0}=e^{\eta}\left(\frac{1+K}{1+K^{\prime}}\right)\left\{K^{\prime} \varsigma^{\prime}(0)-\delta_{r}^{\prime}\left(\frac{\partial \varsigma^{\prime}}{\partial x}\right)_{0}\right\}
$$

The problem given by Eqs. (17), (20), (28) and (33) can be solved as indicated in the Appendix A introducing the variable changes:

$$
\begin{aligned}
& s=\frac{x}{2 \sqrt{D t}} \\
& s^{\prime}=\frac{x}{2 \sqrt{D^{\prime} t}} \\
& \chi=\sqrt{\kappa t}
\end{aligned}
$$

and the following expressions are obtained for the concentration profiles of the pseudo-species $\varsigma$ and $\varsigma^{\prime}$ :

$$
\begin{aligned}
& \varsigma=\varsigma^{*}+\frac{\varsigma^{*}}{1+K^{\prime} \gamma e^{\eta}\left(\frac{1+K}{1+K^{\prime}}\right)} \sum_{j=1}^{\infty}\left\{\frac{(-1)^{\mathrm{j}} \chi_{\mathrm{CEC}}^{\mathrm{j}}}{\prod_{l=1}^{j} p_{l}} \sum_{m=0}^{\infty}\left(d_{j, m}-p_{j} e_{j, m} s\right) \mathrm{s}^{m}\right\} \\
& \varsigma^{\prime}=-\gamma \frac{\varsigma^{*}}{1+K^{\prime} \gamma e^{\eta}\left(\frac{1+K}{1+K^{\prime}}\right)} \sum_{j=1}^{\infty}\left\{\frac{(-1)^{\mathrm{j}} \chi_{\mathrm{CEC}}^{\mathrm{j}}}{\prod_{l=1}^{j} p_{l}} \sum_{m=0}^{\infty}\left(d_{j, m}-p_{j} e_{j, m} s^{\prime}\right) \mathrm{s}^{\prime m}\right\}
\end{aligned}
$$

such that:

$$
\sqrt{D} \varsigma(0)+\sqrt{D^{\prime}} \varsigma^{\prime}(0)=\sqrt{D} \varsigma^{*}
$$

with: 


$$
\chi_{\mathrm{CEC}}=2 \sqrt{\kappa t}\left(\frac{1+K^{\prime} \gamma e^{\eta}\left(\frac{1+K}{1+K^{\prime}}\right)}{K+\sqrt{\frac{\kappa}{\kappa^{\prime}}} \gamma e^{\eta}\left(\frac{1+K}{1+K^{\prime}}\right)}\right)
$$

and:

$$
\begin{gathered}
d_{j, 0}=1 ; d_{j, m+2}=d_{j, m} \frac{2(j-m)}{(m+1)(m+2)} \\
e_{j, 0}=1 ; e_{j, m+2}=e_{j, m} \frac{2(j-m-1)}{(m+2)(m+3)} \\
p_{j}=\frac{2 \Gamma\left(1+\frac{j}{2}\right)}{\Gamma\left(\frac{1+j}{2}\right)}
\end{gathered}
$$

with $p_{0}=2 / \sqrt{\pi}$ and $p_{j} p_{j+1}=2(j+1)$.

The surface gradients of pseudo-species $\varsigma$ and $\zeta^{\prime}$ can be easily obtained from Eqs. (35)

$$
\begin{aligned}
& \left(\frac{\partial \varsigma}{\partial x}\right)_{0}=\left(\frac{\partial s}{\partial x}\right)\left(\frac{\partial \varsigma}{\partial s}\right)_{0}=\frac{1}{\sqrt{\pi D t}} \frac{\varsigma^{*}}{1+K^{\prime} \gamma e^{\eta}\left(\frac{1+K}{1+K^{\prime}}\right)} F\left(\chi_{\mathrm{CEC}}\right) \\
& \left(\frac{\partial \varsigma^{\prime}}{\partial x}\right)_{0}=\left(\frac{\partial s^{\prime}}{\partial x}\right)\left(\frac{\partial \varsigma^{\prime}}{\partial s^{\prime}}\right)_{0}=-\frac{1}{\sqrt{\pi D^{\prime} t}} \frac{\gamma \varsigma^{*}}{1+K^{\prime} \gamma e^{\eta}\left(\frac{1+K}{1+K^{\prime}}\right)} F\left(\chi_{\mathrm{CEC}}\right)
\end{aligned}
$$

such that the solutions for $\phi$ and $\phi^{\prime}$ are (see Eqs. (27), (29) and (30)):

$$
\begin{aligned}
& \phi=K \frac{\delta_{r}}{\delta} \frac{\varsigma^{*}}{1+K^{\prime} \gamma e^{\eta}\left(\frac{1+K}{1+K^{\prime}}\right)} F\left(\chi_{\mathrm{CEC}}\right) \exp \left(-\frac{x}{\delta_{\mathrm{r}}}\right) \\
& \phi^{\prime}=\left(\frac{\delta_{r}^{\prime}}{\delta^{\prime}}\right) \gamma \frac{\varsigma^{*}}{1+K^{\prime} \gamma e^{\eta}\left(\frac{1+K}{1+K^{\prime}}\right)} F\left(\chi_{\mathrm{CEC}}\right) \exp \left(-\frac{x}{\delta_{\mathrm{r}}^{\prime}}\right)
\end{aligned}
$$

with:

$$
F(x)=\sum_{j=0}^{\infty} \frac{(-1)^{j} x^{j+1}}{\prod_{l=0}^{j} p_{l}}=\sqrt{\pi} \frac{x}{2} \exp \left(\frac{x}{2}\right)^{2} \operatorname{erfc}\left(\frac{x}{2}\right)
$$

and $\delta=\sqrt{\pi D t}$ and $\delta^{\prime}=\sqrt{\pi D^{\prime} t}$ correspond to the thickness of the linear diffusion layer. From the above expressions, the concentration profiles of the species can be finally calculated considering the following relationships: 


$$
\begin{aligned}
& c_{\mathrm{O}}=\frac{\varsigma-\phi}{1+\mathrm{K}} \\
& c_{\mathrm{Z}}=\frac{K \varsigma+\phi}{1+\mathrm{K}} \\
& c_{\mathrm{R}}=\frac{K^{\prime} \varsigma^{\prime}+\phi^{\prime}}{1+\mathrm{K}^{\prime}} \\
& c_{\mathrm{Z}^{\prime}}=\frac{\varsigma^{\prime}-\phi^{\prime}}{1+\mathrm{K}^{\prime}}
\end{aligned}
$$

Regarding the current-potential response of the CEC mechanism, this is immediately obtained from the surface gradient of species $\varsigma$ (Eq. (40)):

$$
I_{\mathrm{CEC}}=F A D \frac{\varsigma^{*}}{\delta} \frac{F\left(\chi_{\mathrm{CEC}}\right)}{1+K^{\prime} \gamma e^{\eta}\left(\frac{1+K}{1+K^{\prime}}\right)}
$$

that under limiting current conditions $\left(e^{\eta} \rightarrow 0\right)$ becomes into:

$$
I_{\mathrm{CEC}}^{\lim }=F A D \frac{\varsigma^{*}}{\delta} F\left(\chi_{\mathrm{CEC}}^{\lim }\right)
$$

with $\chi_{\mathrm{CEC}}^{\lim }=\frac{2 \sqrt{\kappa t}}{K}$.

Considering Eq. (1), the following explicit expressions for the absorbance response when the light beam is incident normal to the electrode surface are obtained by introducing Eqs. (35) , (41), (43) and (44) in Eq. (1): 


$$
\begin{aligned}
& \frac{\mathrm{A}_{\mathrm{O}, \mathrm{N}}(\lambda, t)}{\varepsilon_{0}(\lambda) \zeta^{*} \delta}=\frac{1}{1+\mathrm{K}}\left\{\frac{2}{\sqrt{\pi}} \frac{1}{1+\mathrm{K}^{\prime} \gamma \mathrm{e}^{\mathrm{n}}\left(\frac{1+\mathrm{K}}{1+\mathrm{K}^{\prime}}\right)} \sum_{\mathrm{j}=1}^{\infty}\left(\frac{(-1)^{\mathrm{j}} \chi_{\mathrm{CEC}}^{\mathrm{j}}}{\prod_{\mathrm{l}=1}^{\mathrm{j}} \mathrm{p}_{\mathrm{l}}} \sum_{\mathrm{m}=0}^{\infty}\left(\frac{d_{\mathrm{j}, \mathrm{m}}}{\mathrm{m}+1}-\frac{e_{\mathrm{j}, \mathrm{m}}}{\mathrm{m}+2} p_{\mathrm{j}} \frac{l}{2 \sqrt{\mathrm{Dt}}}\right)\left(\frac{l}{2 \sqrt{\mathrm{Dt}}}\right)^{\mathrm{m}+1}\right)+\right. \\
& \left.+\frac{l}{\delta}-K\left(\frac{\delta_{\mathrm{r}}}{\delta}\right)^{2} \frac{1}{1+\mathrm{K}^{\prime} \gamma \mathrm{e}^{\mathrm{n}}\left(\frac{1+\mathrm{K}}{1+\mathrm{K}^{\prime}}\right)} \mathrm{F}\left(\chi_{\mathrm{CEC}}\right)\right\} \\
& \frac{\mathrm{A}_{\mathrm{Z}, \mathrm{N}}(\lambda, t)}{\varepsilon_{\mathrm{Z}}(\lambda) \zeta^{*} \delta}=\frac{K}{1+K}\left\{\frac{2}{\sqrt{\pi}} \frac{1}{1+\mathrm{K}^{\prime} \gamma \mathrm{e}^{\eta}\left(\frac{1+\mathrm{K}}{1+\mathrm{K}^{\prime}}\right)} \sum_{\mathrm{j}=1}^{\infty}\left(\frac{(-1)^{\mathrm{j}} \chi_{\mathrm{CEC}}^{\mathrm{j}}}{\prod_{\mathrm{l}=1}^{\mathrm{j}} \mathrm{p}_{\mathrm{l}}} \sum_{\mathrm{m}=0}^{\infty}\left(\frac{d_{\mathrm{j}, \mathrm{m}}}{\mathrm{m}+1}-\frac{e_{\mathrm{j}, \mathrm{m}}}{\mathrm{m}+2} p_{\mathrm{j}} \frac{l}{2 \sqrt{\mathrm{Dt}}}\right)\left(\frac{l}{2 \sqrt{\mathrm{Dt}}}\right)^{\mathrm{m}+1}\right)+\right. \\
& \left.+\frac{l}{\delta}+\left(\frac{\delta_{\mathrm{r}}}{\delta}\right)^{2} \frac{1}{1+\mathrm{K}^{\prime} \gamma \mathrm{e}^{\mathrm{\eta}}\left(\frac{1+\mathrm{K}}{1+\mathrm{K}^{\prime}}\right)} \mathrm{F}\left(\chi_{\mathrm{CEC}}\right)\right\} \\
& \frac{\mathrm{A}_{\mathrm{R}, \mathrm{N}}(\lambda, t)}{\varepsilon_{\mathrm{R}}(\lambda) \zeta^{*} \delta^{\prime}}=\frac{\gamma}{1+K^{\prime}}\left\{-\frac{2}{\sqrt{\pi}} \frac{K^{\prime}}{1+\mathrm{K}^{\prime} \gamma \mathrm{e}^{\mathrm{\eta}}\left(\frac{1+\mathrm{K}}{1+\mathrm{K}^{\prime}}\right)} \sum_{\mathrm{j}=1}^{\infty}\left(\frac{(-1)^{j} \chi_{\mathrm{CEC}}^{j}}{\prod_{\mathrm{l}=1}^{j} \mathrm{p}_{\mathrm{l}}} \sum_{\mathrm{m}=0}^{\infty}\left(\frac{d_{\mathrm{j}, \mathrm{m}}}{\mathrm{m}+1}-\frac{e_{\mathrm{j}, \mathrm{m}}}{\mathrm{m}+2} p_{\mathrm{j}} \frac{l}{2 \sqrt{\mathrm{D}^{\prime} \mathrm{t}}}\right)\left(\frac{l}{2 \sqrt{\mathrm{D}^{\prime} \mathrm{t}}}\right)^{\mathrm{m}+1}\right)+\right. \\
& \left.+\left(\frac{\delta_{\mathrm{r}}^{\prime}}{\delta^{\prime}}\right)^{2} \frac{1}{1+\mathrm{K}^{\prime} \gamma \mathrm{e}^{\eta}\left(\frac{1+\mathrm{K}}{1+\mathrm{K}^{\prime}}\right)} \mathrm{F}\left(\chi_{\mathrm{CEC}}\right)\right\} \\
& \frac{\mathrm{A}_{\mathrm{Z}^{\prime}, \mathrm{N}}(\lambda, t)}{\varepsilon_{\mathrm{Z}^{\prime}}(\lambda) \zeta^{*} \delta^{\prime}}=\frac{\gamma}{1+K^{\prime}}\left\{-\frac{2}{\sqrt{\pi}} \frac{1}{1+\mathrm{K}^{\prime} \gamma \mathrm{e}^{\mathrm{\eta}}\left(\frac{1+\mathrm{K}}{1+\mathrm{K}^{\prime}}\right)} \sum_{\mathrm{j}=1}^{\infty}\left(\frac{(-1)^{j} \chi_{\mathrm{CEC}}^{j}}{\prod_{\mathrm{l}=1}^{\mathrm{j}} \mathrm{p}_{\mathrm{l}}} \sum_{\mathrm{m}=0}^{\infty}\left(\frac{d_{\mathrm{j}, \mathrm{m}}}{\mathrm{m}+1}-\frac{e_{\mathrm{j}, \mathrm{m}}}{\mathrm{m}+2} p_{\mathrm{j}} \frac{l}{2 \sqrt{\mathrm{D}^{\prime} \mathrm{t}}}\right)\left(\frac{l}{2 \sqrt{\mathrm{D}^{\prime} \mathrm{t}}}\right)^{\mathrm{m}+1}\right)-\right. \\
& \left.-\left(\frac{\delta_{\mathrm{r}}^{\prime}}{\delta^{\prime}}\right)^{2} \frac{1}{1+\mathrm{K}^{\prime} \gamma \mathrm{e}^{\eta}\left(\frac{1+\mathrm{K}}{1+\mathrm{K}^{\prime}}\right)} \mathrm{F}\left(\chi_{\mathrm{CEC}}\right)\right\}
\end{aligned}
$$

Note that for values $l / 2 \sqrt{D^{()} t}>2$ (as it is the case here) it is found that the value of the sum in $m$ does not change significantly; hence, in order to avoid convergence issues of this sum, the term $l / 2 \sqrt{D^{()} t}$ in Eqs. (47)-(50) can be replaced by 2 without compromising the accuracy of the results. 
The SEC response in the parallel beam configuration, $\mathrm{A}_{i, \mathrm{P}}^{\mathrm{kss}}(\lambda, t)$, can be calculated by numerical integration of Eq. (2) with $c_{i}(x, t)$ being given by Eqs. (43)-(44). By comparison with rigorous solutions, it has been found that the theoretical treatment developed above leads to accurate values for $\left(k_{1}+k_{2}\right) t>5$ and/or $\left(k_{1}^{\prime}+k_{2}^{\prime}\right) t>5[26,27]$.

\subsection{Diffusive-kinetic steady state solution}

For relatively fast chemical kinetics $\left(2 \sqrt{\kappa t} / \mathrm{K}>10, \kappa^{\prime} t>6\right)$, the diffusive-kinetic steady state (dkss) theoretical treatment can be used to obtain simpler, closed-form expressions for the concentration profiles and the absorbance response [23]. Following the dkss approach, the form of the solutions for $\phi$ and $\phi^{\prime}$ are assumed to be given by Eqs. (27) and, additionally, the mathematical form of $\varsigma^{d k s s}$ and $\varsigma^{\prime d k s s}$ are supposed to be equivalent to that of a species only subject to diffusion:

$$
\begin{aligned}
& \varsigma^{d k s s}=\varsigma^{*}+\left(\varsigma^{d k s s}(0)-\varsigma^{*}\right) \operatorname{erfc}\left(\frac{x}{2 \sqrt{D t}}\right) \\
& \varsigma^{\prime d k s s}=\varsigma^{\prime d k s s}(0) \operatorname{erfc}\left(\frac{x}{2 \sqrt{D^{\prime} t}}\right)
\end{aligned}
$$

so that:

$$
\begin{aligned}
& \left(\frac{\partial \varsigma^{d k s s}}{\partial x}\right)_{0}=\frac{\varsigma^{*}-\varsigma^{d k s s}(0)}{\delta} \\
& \left(\frac{\partial \varsigma^{\prime d k s s}}{\partial x}\right)_{0}=\frac{-\varsigma^{d k s s}(0)}{\delta^{\prime}}
\end{aligned}
$$

Taking the above into account and applying the surface boundary condition (33), the following expressions are obtained for the surface values $\varsigma^{d k s s}(0)$ and $\varsigma^{\prime d k s s}(0)$ : 


$$
\begin{gathered}
\varsigma^{d k s s}(0)=\varsigma^{*} \frac{K \frac{\delta_{\mathrm{r}}}{\delta}+\left(\frac{1+K}{1+K^{\prime}}\right) \gamma \mathrm{e}^{\eta}\left(\frac{\delta_{\mathrm{r}}^{\prime}}{\delta^{\prime}}+K^{\prime}\right)}{1+K \frac{\delta_{\mathrm{r}}}{\delta}+\left(\frac{1+K}{1+K^{\prime}}\right) \gamma \mathrm{e}^{\eta}\left(\frac{\delta_{\mathrm{r}}^{\prime}}{\delta^{\prime}}+K^{\prime}\right)} \\
\varsigma^{\prime d k s s}(0)=\varsigma^{*} \frac{\gamma}{1+K \frac{\delta_{\mathrm{r}}}{\delta}+\left(\frac{1+K}{1+K^{\prime}}\right) \gamma \mathrm{e}^{\eta}\left(\frac{\delta_{\mathrm{r}}^{\prime}}{\delta^{\prime}}+K^{\prime}\right)}
\end{gathered}
$$

and also for $\phi^{d k s s}(0)$ and $\phi^{\prime d k s s}(0)$ (see Eqs. (29), (30) and (53)):

$$
\begin{aligned}
& \phi^{d k s s}(0)=K \frac{\delta_{r}}{\delta}\left(\frac{\varsigma^{*}}{1+K \frac{\delta_{\mathrm{r}}}{\delta}+\left(\frac{1+K}{1+K^{\prime}}\right) \gamma \mathrm{e}^{\eta}\left(\frac{\delta_{\mathrm{r}}^{\prime}}{\delta^{\prime}}+K^{\prime}\right)}\right) \\
& \phi^{\prime d k s s}(0)=\gamma\left(\frac{\delta_{r}^{\prime}}{\delta^{\prime}}\right)\left(\frac{\varsigma^{*}}{1+K \frac{\delta_{\mathrm{r}}}{\delta}+\left(\frac{1+K}{1+K^{\prime}}\right) \gamma \mathrm{e}^{\eta}\left(\frac{\delta_{\mathrm{r}}^{\prime}}{\delta^{\prime}}+K^{\prime}\right)}\right)
\end{aligned}
$$

Finally, the concentration profiles of the species $O, R, Z$, and $Z^{\prime}$ are derived by introducing Eqs.

(27), (51), (53) and (54) in the relationships (43) and (44):

$$
\begin{aligned}
& c_{\mathrm{O}}^{\mathrm{dkss}}=\frac{\varsigma^{*}}{1+K}\left\{1-\left(\frac{\operatorname{erfc}\left(\frac{x}{2 \sqrt{\mathrm{Dt}}}\right)+K \frac{\delta_{\mathrm{r}}}{\delta} \exp \left(-\frac{\mathrm{x}}{\delta_{\mathrm{r}}}\right)}{1+K \frac{\delta_{\mathrm{r}}}{\delta}+\left(\frac{1+K}{1+K^{\prime}}\right) \gamma \mathrm{e}^{\eta}\left(\frac{\delta_{\mathrm{r}}^{\prime}}{\delta^{\prime}}+K^{\prime}\right)}\right)\right\} \\
& c_{\mathrm{Z}}^{\mathrm{dkss}}=\frac{\varsigma^{*} K}{1+K}\left\{1-\left(\frac{\operatorname{erfc}\left(\frac{x}{2 \sqrt{\mathrm{Dt}}}\right)-\frac{\delta_{\mathrm{r}}}{\delta} \exp \left(-\frac{\mathrm{x}}{\delta_{\mathrm{r}}}\right)}{1+K \frac{\delta_{\mathrm{r}}}{\delta}+\left(\frac{1+K}{1+K^{\prime}}\right) \gamma \mathrm{e}^{\eta}\left(\frac{\delta_{\mathrm{r}}^{\prime}}{\delta^{\prime}}+K^{\prime}\right)}\right)\right\} \\
& c_{\mathrm{R}}^{\mathrm{dkss}}=\frac{\varsigma^{*}}{1+\mathrm{K}^{\prime}} \gamma\left\{\frac{K^{\prime} \operatorname{erfc}\left(\frac{x}{2 \sqrt{\mathrm{D}^{\prime} \mathrm{t}}}\right)+\frac{\delta_{\mathrm{r}}^{\prime}}{\delta^{\prime}} \exp \left(-\frac{\mathrm{x}}{\delta_{\mathrm{r}}^{\prime}}\right)}{1+\mathrm{K} \frac{\delta_{\mathrm{r}}}{\delta}+\left(\frac{1+\mathrm{K}}{1+\mathrm{K}^{\prime}}\right) \gamma \mathrm{e}^{\eta}\left(\frac{\delta_{\mathrm{r}}^{\prime}}{\delta^{\prime}}+\mathrm{K}^{\prime}\right)}\right\} \\
& c_{\mathrm{Z}^{\prime}}^{\mathrm{dkss}}=\frac{\varsigma^{*}}{1+\mathrm{K}^{\prime}} \gamma\left\{\frac{\operatorname{erfc}\left(\frac{x}{2 \sqrt{\mathrm{D}^{\prime} \mathrm{t}}}\right)-\frac{\delta_{\mathrm{r}}^{\prime}}{\delta^{\prime}} \exp \left(-\frac{\mathrm{x}}{\delta_{\mathrm{r}}^{\prime}}\right)}{1+\mathrm{K} \frac{\delta_{\mathrm{r}}}{\delta}+\left(\frac{1+\mathrm{K}}{1+\mathrm{K}^{\prime}}\right) \gamma \mathrm{e}^{\eta}\left(\frac{\delta_{\mathrm{r}}^{\prime}}{\delta^{\prime}}+\mathrm{K}^{\prime}\right)}\right\}
\end{aligned}
$$


and, from Eq. (1) with $l>>\delta, \delta^{\prime}$ and $l>>\delta_{\mathrm{r}}, \delta_{\mathrm{r}}^{\prime}$, the contribution of each species to the absorbance response in the normal beam configuration is deduced:

$$
\begin{aligned}
& \frac{\mathrm{A}_{\mathrm{O}, \mathrm{N}}^{\mathrm{dkss}}(\lambda, t)}{\varepsilon_{\mathrm{O}}(\lambda) \varsigma^{*} \delta}=\frac{1}{1+K}\left\{\frac{l}{\delta}-\left(\frac{\frac{2}{\pi}+K\left(\frac{\delta_{\mathrm{r}}}{\delta}\right)^{2}}{1+K \frac{\delta_{\mathrm{r}}}{\delta}+\left(\frac{1+K}{1+K^{\prime}}\right) \gamma \mathrm{e}^{\eta}\left(\frac{\delta_{\mathrm{r}}^{\prime}}{\delta^{\prime}}+K^{\prime}\right)}\right)\right\} \\
& \frac{\mathrm{A}_{\mathrm{ZN}}^{\mathrm{dkss}}(\lambda, t)}{\varepsilon_{\mathrm{Z}}(\lambda) \varsigma^{*} \delta}=\frac{K}{1+K}\left\{\frac{l}{\delta}-\left(\frac{\frac{2}{\pi}-\left(\frac{\delta_{\mathrm{r}}}{\delta}\right)^{2}}{1+K \frac{\delta_{\mathrm{r}}}{\delta}+\left(\frac{1+K}{1+K^{\prime}}\right) \gamma \mathrm{e}^{\eta}\left(\frac{\delta_{\mathrm{r}}^{\prime}}{\delta^{\prime}}+K^{\prime}\right)}\right)\right\} \\
& \frac{\mathrm{A}_{\mathrm{R}, \mathrm{N}}^{\mathrm{dkss}}(\lambda, t)}{\varepsilon_{\mathrm{R}}(\lambda) \varsigma^{*} \delta^{\prime}}=\frac{\gamma}{1+K^{\prime}}\left(\frac{\frac{2}{\pi} K^{\prime}+\left(\frac{\delta_{\mathrm{r}}^{\prime}}{\delta^{\prime}}\right)^{2}}{1+K \frac{\delta_{\mathrm{r}}}{\delta}+\left(\frac{1+K}{1+K^{\prime}}\right) \gamma \mathrm{e}^{\eta}\left(\frac{\delta_{\mathrm{r}}^{\prime}}{\delta^{\prime}}+K^{\prime}\right)}\right) \\
& \frac{\mathrm{A}_{\mathrm{Z}^{\prime} \mathrm{N}}^{\mathrm{dkss}}(\lambda, t)}{\varepsilon_{\mathrm{Z}^{\prime}}(\lambda) \varsigma^{*} \delta^{\prime}}=\frac{\gamma}{1+K^{\prime}}\left(\frac{\frac{2}{\pi}-\left(\frac{\delta_{\mathrm{r}}^{\prime}}{\delta^{\prime}}\right)^{2}}{1+K \frac{\delta_{\mathrm{r}}}{\delta}+\left(\frac{1+K}{1+K^{\prime}}\right) \gamma \mathrm{e}^{\eta}\left(\frac{\delta_{\mathrm{r}}^{\prime}}{\delta^{\prime}}+K^{\prime}\right)}\right)
\end{aligned}
$$

With regard to the response in the parallel beam configuration, $\mathrm{A}_{i, \mathrm{P}}^{\mathrm{dkss}}(\lambda, t)$, this can be calculated numerically from Eqs. (2) and (55)-(56). 


\section{Results and Discussion}

With the expressions given in Section 2, the concentration profiles of all the species in the CEC mechanism when applying a potential pulse perturbation at a macroelectrode can be calculated as well as the SEC response in both normal and parallel beam configurations. Given the generality of the reaction scheme considered, the analytical solutions cover situations where none, one or both electroactive species take part in homogeneous chemical reactions (CEC, EC, CE and E mechanisms) and where one or more species are photoactive. In the following sections, the influence of the chemical rate and equilibrium constants will be examined for some specific situations of interest. Limiting current conditions will be considered in all cases (i.e., $e^{\eta} \rightarrow 0$ ) since this enables us to avoid the influence of uncertainties in the electrode kinetics and formal potential, the SEC response being only a function of the characteristics of the homogeneous chemical reactions.

In Figure 1, the concentration profiles of all the species are plotted from which the reaction layer can be immediately identified as the region close to the electrode surface where equilibrium conditions are broken (that is, where $c_{\mathrm{Z}} \neq c_{\mathrm{O}}$ and $c_{\mathrm{Z}^{\prime}} \neq c_{\mathrm{R}}$ in Figure 1a since $\left.K=K^{\prime}=1\right)$. Note that such a region is larger for reactants than for products in Figure 1a due to the slower kinetics of the preceding chemical reaction: $\kappa^{\prime}=10 \kappa$. In Figure $1 b$, the constant $K^{\prime}$ is assumed to be very large $(=100)$ such that the formation of species $Z^{\prime}$ is negligible and the system behaves as a CE-like mechanism. On the other hand, the EC mechanism corresponds to the limit case where the constant $\mathrm{K}$ is very small $(=0.01$ in Figure $1 \mathrm{c})$. 
$\mathrm{Z} \stackrel{\kappa, K}{\rightleftarrows} \mathrm{O} ; \mathrm{O}+e^{-} \rightleftarrows \mathrm{R} ; \quad \mathrm{R} \stackrel{\kappa^{\prime}, K^{\prime}}{\rightleftarrows} \mathrm{Z}^{\prime}$

(a) CEC $\mathrm{K}=2 \mathrm{~s}^{-1}, \mathrm{~K}^{\prime}=20 \mathrm{~s}^{-1} ; \mathrm{K}=\mathrm{K}^{\prime}=1 ; t=1 \mathrm{~s}$

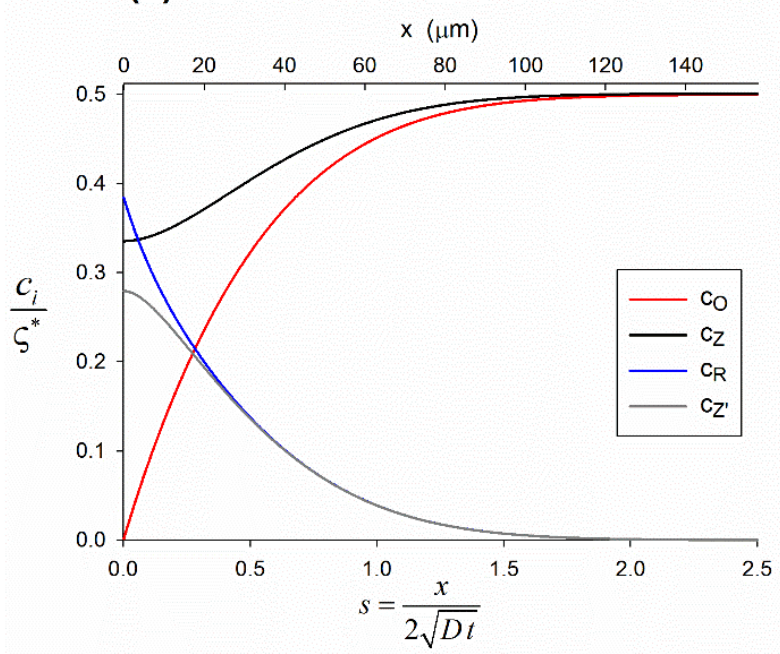

(b) CE $\kappa=5 \mathrm{~s}^{-1} ; K=1, K^{\prime}=10^{2} ; t=1 \mathrm{~s}$

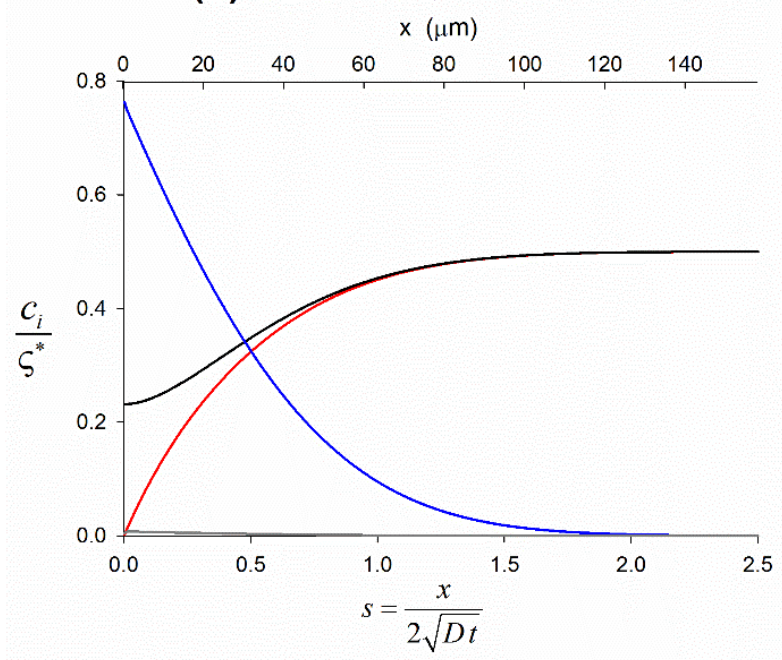

(c) EC $\kappa^{\prime}=5 \mathrm{~s}^{-1} ; \quad K^{\prime}=1, K=0.01 ; t=1 \mathrm{~s}$

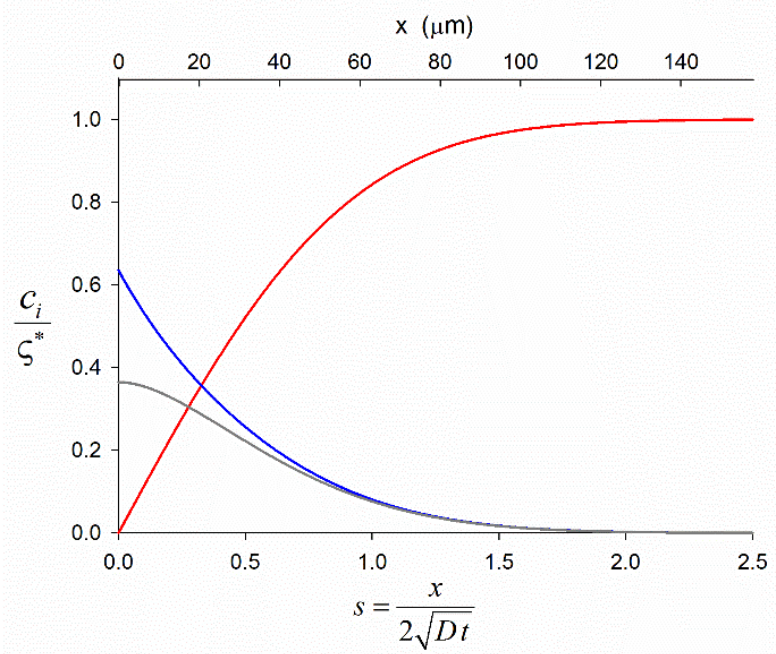

Figure 1. Concentration profiles (Eqs. (35) and (41), (43) and (44)) of the CEC mechanism under different conditions of chemical kinetics and thermodynamics; note that cases b) and c) correspond to CE-like and EC-like mechanisms, respectively. $D=D^{\prime}$, limiting current conditions $\left(\mathrm{e}^{\eta} \rightarrow 0\right.$ ) 


\subsection{E mechanism}

The case of simple charge transfer processes (i.e., the E mechanism) where the species $\mathrm{O}$ and $\mathrm{R}$ do not undergo any chemical process in solution can be derived from the solutions obtained for the CEC mechanism (Eqs. (43), (44), (47)-(50) and Eqs. (55)-(58)) by making $K \rightarrow 0$ and $K^{\prime}>>1$. Thus, the following expressions are obtained for the concentration profiles $[8,25]$ :

$$
\begin{aligned}
& c_{\mathrm{O}}^{\mathrm{E}}=c_{\mathrm{O}}^{*}\left\{1-\frac{1}{1+\gamma \mathrm{e}^{\eta}} \operatorname{erfc}\left(\frac{x}{2 \sqrt{\mathrm{Dt}}}\right)\right\} \\
& c_{\mathrm{R}}^{\mathrm{E}}=\frac{c_{\mathrm{O}}^{*} \gamma}{1+\gamma \mathrm{e}^{\eta}} \operatorname{erfc}\left(\frac{x}{2 \sqrt{\mathrm{D}^{\prime} \mathrm{t}}}\right)
\end{aligned}
$$

and for the normal-beam SEC signal for any applied potential (see Eq. (4)):

$$
\begin{aligned}
& \frac{\mathrm{A}_{\mathrm{O}, \mathrm{N}}^{\mathrm{E}}(\lambda, t)}{\varepsilon_{\mathrm{O}}(\lambda) \varsigma^{*} \delta}=\frac{l}{\delta}-\frac{2}{\pi} \frac{1}{1+\gamma \mathrm{e}^{\eta}} \\
& \frac{\mathrm{A}_{\mathrm{R}, \mathrm{N}}^{\mathrm{E}}(\lambda, t)}{\varepsilon_{\mathrm{R}}(\lambda) \varsigma^{*} \delta^{\prime}}=\frac{2}{\pi} \frac{\gamma}{1+\gamma \mathrm{e}^{\eta}}
\end{aligned}
$$

\subsection{CE-like mechanisms}

$$
\begin{aligned}
& Z \underset{k_{2}}{\stackrel{k_{1}}{\rightleftarrows} O} \quad ; K=c_{Z}^{e q} / c_{O}^{e q}=k_{2} / k_{1} \\
& O+e^{-} \rightleftarrows R \quad ; E^{0^{\prime}}
\end{aligned}
$$

The CE mechanism is another particular case of the CEC scheme where $K^{\prime}>>1$; hence, the analytical expressions (47)-(50) for the absorbance response in the normal beam configuration become into: 


$$
\begin{aligned}
\frac{\mathrm{A}_{\mathrm{O}, \mathrm{N}}^{\mathrm{CE}}(\lambda, t)}{\varepsilon_{\mathrm{O}}(\lambda) \zeta^{*} \delta}=\frac{1}{1+\mathrm{K}}\left\{\frac{1}{1+\gamma \mathrm{e}^{\mathrm{\eta}}(1+\mathrm{K})} \frac{2}{\sqrt{\pi}} \sum_{\mathrm{j}=1}^{\infty}\left(\frac{(-1)^{\mathrm{j}} \chi_{\mathrm{CE}}^{\mathrm{j}}}{\prod_{\mathrm{l}=1}^{\mathrm{j}} \mathrm{p}_{1}} \sum_{\mathrm{m}=0}^{\infty}\left(\frac{d_{\mathrm{j}, \mathrm{m}}}{\mathrm{m}+1}-\frac{e_{\mathrm{j}, \mathrm{m}}}{\mathrm{m}+2} p_{\mathrm{j}} \frac{l}{2 \sqrt{\mathrm{Dt}}}\right)\left(\frac{l}{2 \sqrt{\mathrm{Dt}}}\right)^{\mathrm{m}+1}\right)+\right. \\
\left.+\frac{l}{\delta}-K\left(\frac{\delta_{\mathrm{r}}}{\delta}\right)^{2} \frac{1}{1+\gamma \mathrm{e}^{\mathrm{\eta}}(1+\mathrm{K})} \mathrm{F}\left(\chi_{\mathrm{CE}}\right)\right\} \\
\frac{\mathrm{A}_{\mathrm{Z}, \mathrm{N}}^{\mathrm{CE}}(\lambda, t)}{\varepsilon_{\mathrm{Z}}(\lambda) \zeta^{*} \delta}=\frac{K}{1+K}\left\{\frac{2}{\sqrt{\pi}} \frac{1}{1+\gamma \mathrm{e}^{\mathrm{\eta}}(1+\mathrm{K})} \sum_{\mathrm{j}=1}^{\infty}\left(\frac{(-1)^{\mathrm{j}} \chi_{\mathrm{CE}}^{\mathrm{j}}}{\prod_{\mathrm{l}=1}^{\mathrm{j}} \mathrm{p}_{1}} \sum_{\mathrm{m}=0}^{\infty}\left(\frac{d_{\mathrm{j}, \mathrm{m}}}{\mathrm{m}+1}-\frac{e_{\mathrm{j}, \mathrm{m}}}{\mathrm{m}+2} p_{\mathrm{j}} \frac{l}{2 \sqrt{\mathrm{Dt}}}\right)\left(\frac{l}{2 \sqrt{\mathrm{Dt}}}\right)^{\mathrm{m}+1}\right)+\right. \\
\left.+\frac{l}{\delta}+\left(\frac{\delta_{\mathrm{r}}}{\delta}\right)^{2} \frac{1}{1+\gamma \mathrm{e}^{\mathrm{n}}(1+\mathrm{K})} \mathrm{F}^{\mathrm{F}}\left(\chi_{\mathrm{CE}}\right)\right\} \\
\frac{\mathrm{A}_{\mathrm{R}, \mathrm{N}}^{\mathrm{CE}}(\lambda, t)}{\varepsilon_{\mathrm{R}}(\lambda) \zeta^{*} \delta^{\prime}}=-\frac{2}{\sqrt{\pi}} \frac{\gamma}{1+\gamma \mathrm{e}^{\mathrm{\eta}}(1+\mathrm{K})} \sum_{\mathrm{j}=1}^{\infty}\left(\frac{(-1)^{j} \chi_{\mathrm{CE}}^{j}}{\prod_{\mathrm{l}=1}^{\mathrm{j}} \mathrm{p}_{1}} \sum_{\mathrm{m}=0}^{\infty}\left(\frac{d_{\mathrm{j}, \mathrm{m}}}{\mathrm{m}+1}-\frac{e_{\mathrm{j}, \mathrm{m}}}{\mathrm{m}+2} p_{\mathrm{j}} \frac{l}{2 \sqrt{\mathrm{D}^{\prime} \mathrm{t}}}\right)\left(\frac{l}{2 \sqrt{\mathrm{D}^{\prime} \mathrm{t}}}\right)^{\mathrm{m}+1}\right)
\end{aligned}
$$

with:

$$
\chi_{\mathrm{CE}}=\frac{2 \sqrt{\kappa t}}{K}\left(1+\gamma e^{\eta}(1+K)\right)
$$

and the dkss solutions (57) and (58) into:

$$
\begin{gathered}
\frac{\mathrm{A}_{\mathrm{O}, \mathrm{N}}^{\mathrm{dks}, \mathrm{CE}}(\lambda, t)}{\varepsilon_{\mathrm{O}}(\lambda) \varsigma^{*} \delta}=\frac{1}{1+K}\left\{\frac{l}{\delta}-\left(\frac{\frac{2}{\pi}+K\left(\frac{\delta_{\mathrm{r}}}{\delta}\right)^{2}}{1+K \frac{\delta_{\mathrm{r}}}{\delta}+(1+K) \gamma \mathrm{e}^{\eta}}\right)\right\} \\
\frac{\mathrm{A}_{\mathrm{Z}, \mathrm{N}}^{\mathrm{dkss}, \mathrm{CE}}(\lambda, t)}{\varepsilon_{\mathrm{Z}}(\lambda) \zeta^{*} \delta}=\frac{K}{1+K}\left\{\frac{l}{\delta}-\left(\frac{\frac{2}{\pi}-\left(\frac{\delta_{\mathrm{r}}}{\delta}\right)^{2}}{1+K \frac{\delta_{\mathrm{r}}}{\delta}+(1+K) \gamma \mathrm{e}^{\eta}}\right)\right\} \\
\frac{\mathrm{A}_{\mathrm{R}, \mathrm{N}}^{\mathrm{dkss}, \mathrm{CE}}(\lambda, t)}{\varepsilon_{\mathrm{R}}(\lambda) \zeta^{*} \delta^{\prime}}=\frac{2}{\pi} \frac{\gamma}{1+K \frac{\delta_{\mathrm{r}}}{\delta}+(1+K) \gamma \mathrm{e}^{\eta}}
\end{gathered}
$$

Figure 2 shows the SEC response of a CE mechanism where species $\mathrm{R}$ is photoactive. As the $K$-value increases, $\mathrm{A}_{\mathrm{R}}(\lambda, t)$ decreases since there is lower 'availability' of the species $\mathrm{O}$ for the electrode reaction and smaller amounts of the species $R$ are generated. Eventually, when 
the interconversion between $Z$ and $O$ is instantaneous $\left(\chi>>1, \delta_{r} \rightarrow 0\right.$ and $e^{\eta} \rightarrow 0$ in Eq. (67) ), the following expression is obtained for $\mathrm{A}_{\mathrm{R}, \mathrm{N}}^{\mathrm{C}_{\mathrm{e}} \mathrm{E}, \lim }(\lambda, t)$

$$
\mathrm{A}_{\mathrm{R}, \mathrm{N}}^{\mathrm{C}_{\mathrm{e}} \mathrm{E}, \lim }(\lambda, t)=2 \sqrt{\frac{D t}{\pi}} \varepsilon_{\mathrm{R}}(\lambda) \varsigma^{*}
$$

which is equivalent to the expression for the E mechanism with the bulk concentration of the electroactive reagent being $\varsigma^{*}$.

It is worth mentioning that for the case considered in Figure 2, the absorbance response can be calculated, apart from the concentration profile (as in Eq. (58)), also from the current response since the only absorbing species is the electrogenerated one that is stable in solution. Hence, as in the case of the E mechanism, the absorbance is proportional to the total charge passed by the electrode reaction:

$$
\mathrm{A}_{\mathrm{R}, \mathrm{N}}^{\mathrm{CE}}(\lambda, t)=\frac{\varepsilon_{R}(\lambda) Q(t)}{F A}=\frac{\varepsilon_{R}(\lambda)}{F A} \int_{0}^{t} I_{\mathrm{CE}, \mathrm{kss}}(t) d t
$$

where the expression for the current-potential-time response of the CE mechanism is given by (see Eq. (45) with $K^{\prime}>>1$ ) [23]:

$$
I_{\mathrm{CE}}(t)=F A \varsigma^{*} \sqrt{D}\left(\frac{1}{1+(1+K) \gamma e^{\eta}}\right) \frac{1}{\sqrt{\pi t}} F\left(\chi_{\mathrm{CE}}\right)
$$

with:

$$
\chi_{\mathrm{CE}}=2\left(1+(1+K) \gamma e^{\eta}\right) \frac{\sqrt{\kappa t}}{K}
$$

Note that in the limit $\kappa>>1$, it holds that $F\left(\chi_{\mathrm{CE}}\right) \rightarrow 1$ and Eq. (69) with $\mathrm{e}^{\eta} \rightarrow 0$ leads to (68). 


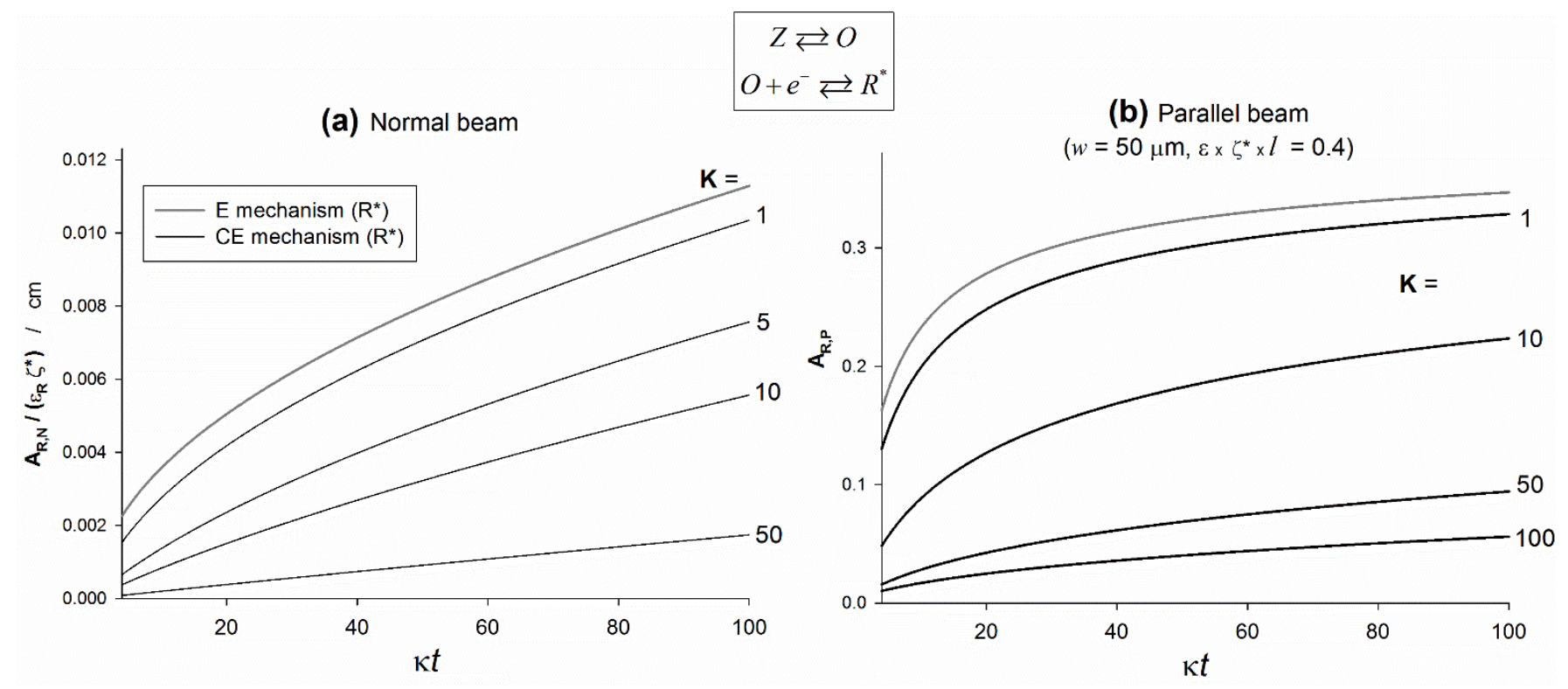

Figure 2. Dimensionless chronoamperometric SEC response of the CE mechanism with $\mathrm{R}$ being the absorbing species as a function of the chemical kinetics $(\kappa)$ and thermodynamics ( $K$ ) plotted using Eq. (64) for the normal configuration and Eqs. (2) and (44) for the parallel one. Other conditions as in Figure 1

Another situation of interest is that where the photoactive species is one of the reactants initially present (i.e., O or Z) [28]. In such situation, the normal beam configuration may not be appropriate to inspect the chemical kinetics since the length of the 'bulk solution' is much larger than the reaction layer so that the absorbance response is scarcely affected by the electrode process (see inset in Figure 4). As shown in Figures 3 and 4, the parallel configuration would be more suitable since by decreasing the $w$-value one can sample a few tens of microns of the solution adjacent to the electrode surface and so the SEC signal is more revealing of the physicochemical dynamics. Thus, the SEC signal does depend significantly on the value of $\kappa$ (see Figure 4 ) and so it enables the determination of the chemical rate constants. 


$$
\begin{gathered}
Z^{*} \rightleftarrows O \\
O+e^{-} \rightleftarrows R
\end{gathered}
$$

(a) Parallel beam

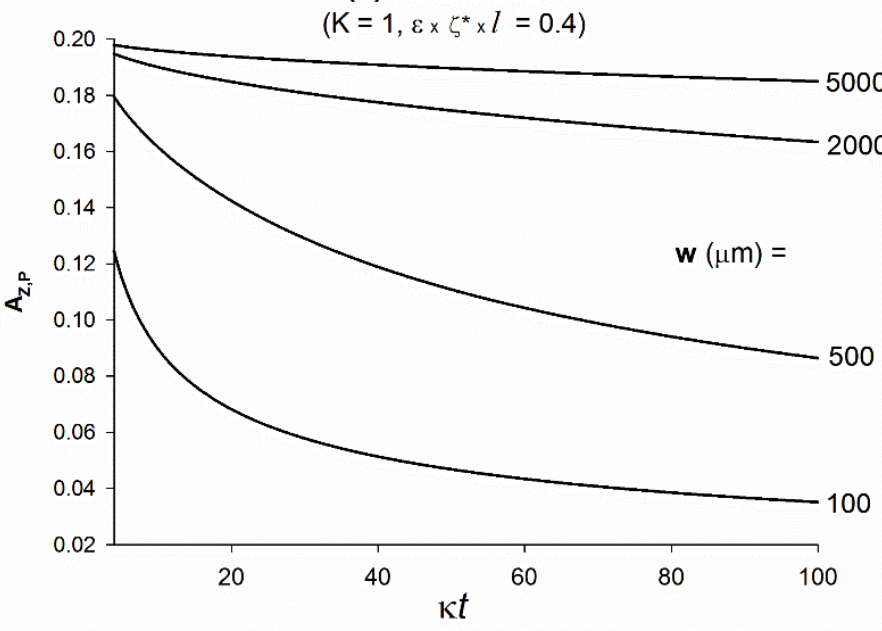

$Z \rightleftarrows O$

$O+e^{-} \rightleftarrows R^{*}$

(b) Parallel beam

$\left(\mathrm{K}=1, \varepsilon \times \zeta^{*} \times l=0.4\right)$

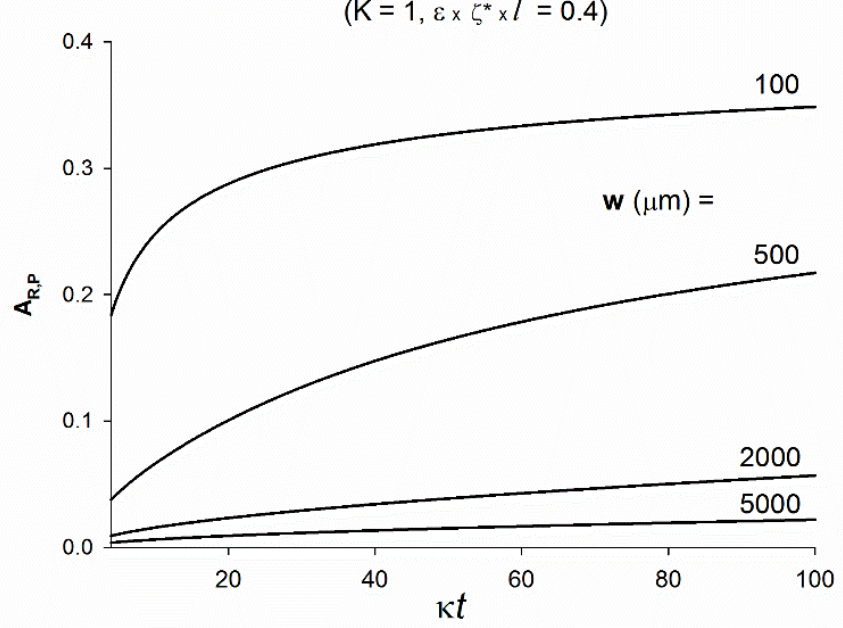

Figure 3. Influence of the $w$-value on the chronoamperometric SEC response of the CE mechanism when (a) Z or (b) R are the absorbing species. Eqs. (2) and (43) and (44). Other conditions as in Figure 1

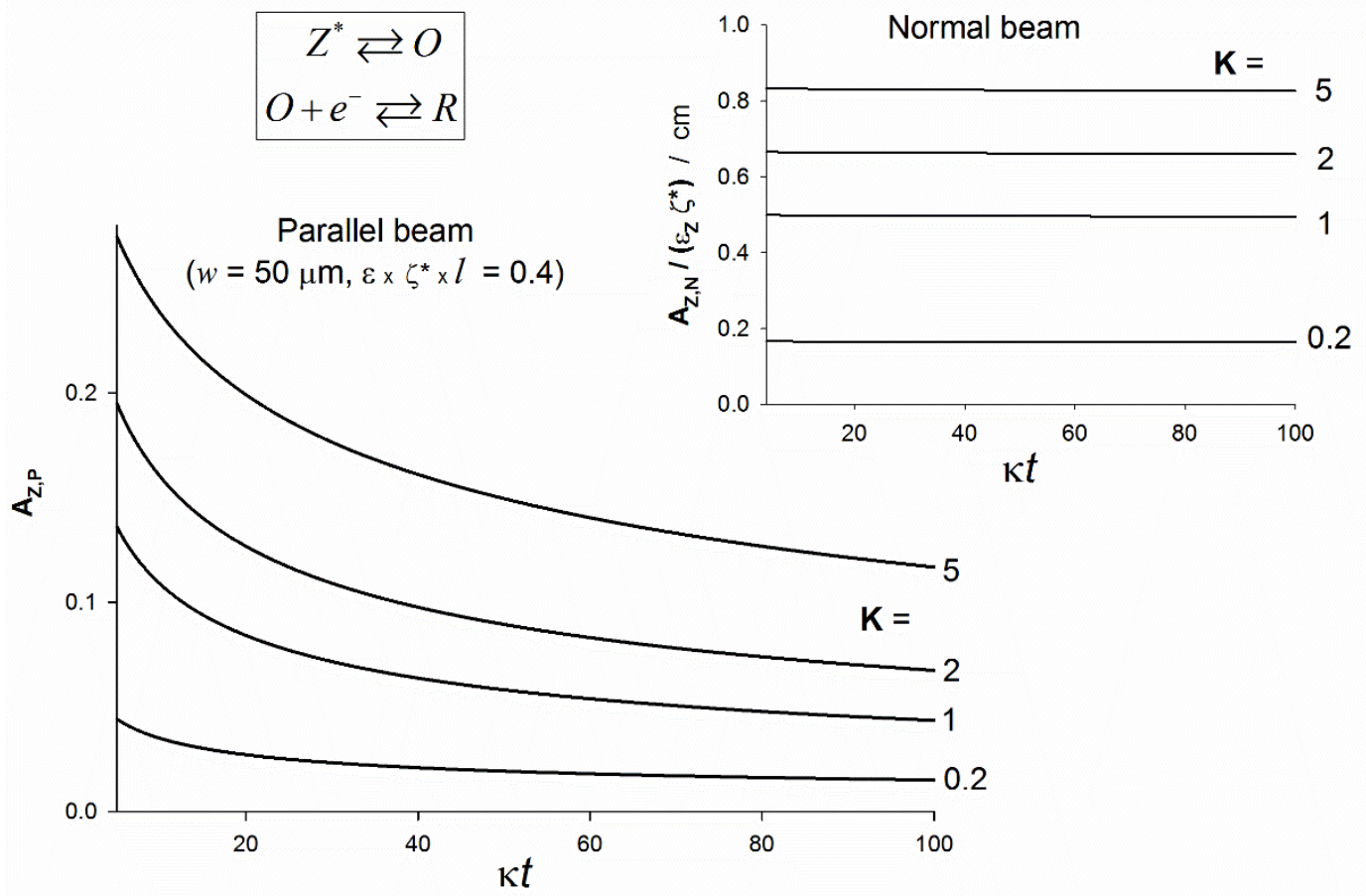

Figure 4. Dimensionless chronoamperometric SEC response of the CE mechanism with Z being the absorbing species as a function of the chemical kinetics $(\chi)$ and thermodynamics $(K)$ plotted using Eq. (63) for the normal configuration (inset) and Eqs. (2) and (43) for the parallel one. Other conditions as in Figure 1 


\subsection{EC-like mechanisms}

$$
\begin{aligned}
O+e^{-} \rightleftarrows & ; E^{0^{\prime}} \\
R \underset{k_{2}^{\prime}}{\rightleftarrows} Z^{\prime} & ; K^{\prime}=c_{R}^{e q} / c_{Z^{\prime}}^{e q}=k_{2}^{\prime} / k_{1}^{\prime}
\end{aligned}
$$

The EC mechanism can be viewed as a particular case of the CEC mechanism where $K \rightarrow 0$; thus, for example, the dkss expressions for the normal beam absorbance transients turn into:

$$
\begin{gathered}
\frac{\mathrm{A}_{\mathrm{O}, \mathrm{N}}^{\mathrm{dks}, \mathrm{EC}}(\lambda, t)}{\varepsilon_{\mathrm{O}}(\lambda) c_{\mathrm{O}}^{*} \delta}=\frac{l}{\delta}-\frac{2}{\pi}\left(\frac{1}{1+\frac{1}{1+K^{\prime}} \gamma \mathrm{e}^{\mathrm{\eta}}\left(\frac{\delta_{\mathrm{r}}^{\prime}}{\delta^{\prime}}+K^{\prime}\right)}\right) \\
\frac{\mathrm{A}_{\mathrm{R}, \mathrm{N}}^{\mathrm{dks}, \mathrm{EC}}(\lambda, t)}{\varepsilon_{\mathrm{R}}(\lambda) c_{\mathrm{O}}^{*} \delta^{\prime}}=\frac{\gamma}{1+K^{\prime}}\left(\frac{\frac{2}{\pi} K^{\prime}+\left(\frac{\delta_{\mathrm{r}}^{\prime}}{\delta^{\prime}}\right)^{2}}{1+\frac{1}{1+K^{\prime}} \gamma \mathrm{e}^{\mathrm{\eta}}\left(\frac{\delta_{\mathrm{r}}^{\prime}}{\delta^{\prime}}+K^{\prime}\right)}\right) \\
\frac{\mathrm{A}_{\mathrm{Z}^{\prime}, \mathrm{N}}^{\mathrm{dks}, \mathrm{EC}}(\lambda, t)}{\varepsilon_{\mathrm{Z}^{\prime}}(\lambda) c_{\mathrm{O}}^{*} \delta^{\prime}}=\frac{\gamma}{1+K^{\prime}}\left(\frac{\frac{2}{\pi}-\left(\frac{\delta_{\mathrm{r}}^{\prime}}{\delta^{\prime}}\right)^{2}}{1+\frac{1}{1+K^{\prime}} \gamma \mathrm{e}^{\eta}\left(\frac{\delta_{\mathrm{r}}^{\prime}}{\delta^{\prime}}+K^{\prime}\right)}\right)
\end{gathered}
$$

This situation is particularly interesting here since the limiting current chronoamperometry of EC-like mechanisms does not inform about the chemical process $[8,25,29]$ and the use of SEC can be very valuable to detect and characterize the latter.

In Figure 5 the SEC response of the EC mechanism is studied when the photoactive species is the electrogenerated one (i.e., species R). As expected, the SEC signal is smaller than in the absence of homogeneous chemical reaction ( $E$ mechanism, grey line) and the decrease is more apparent as the chemical kinetics is faster (larger $\kappa^{\prime}$-values) and the $K^{\prime}$-value decreases. In the limit of very fast chemical kinetics $\left(\kappa^{\prime}>>1\right)$, chemical equilibrium conditions hold and the normal configuration absorbance is easily obtained by making $\delta_{\mathrm{r}}^{\prime} \rightarrow 0$ in Eqs. (73) and (74) ; for example, the response under limiting current conditions when $\mathrm{R}$ is the photoactive species is given by (see Eq. (74) with $\mathrm{e}^{\eta} \rightarrow 0$ and $\delta_{\mathrm{r}}^{\prime} \rightarrow 0$ ): 


$$
\mathrm{A}_{\mathrm{R}, \mathrm{N}}^{\mathrm{EC}}, \lim (\lambda, t)=c_{\mathrm{O}}^{*} \varepsilon_{\mathrm{R}}(\lambda) 2 \sqrt{\frac{D^{\prime} t}{\pi}} \frac{K^{\prime}}{1+K^{\prime}}
$$

The $\mathrm{A}_{\mathrm{R}, \mathrm{N}}^{\mathrm{EC}_{\mathrm{eq}} \text {,lim }}$-value obviously becomes null when the chemical equilibrium is displaced towards the non-absorbing species $\mathrm{Z}\left(K^{\prime} \rightarrow 0\right)$, whereas it is coincident with the response of the $\mathrm{E}$ mechanism (Eq. (4)) in the opposite limit ( $\left.K^{\prime}>>1\right)$.

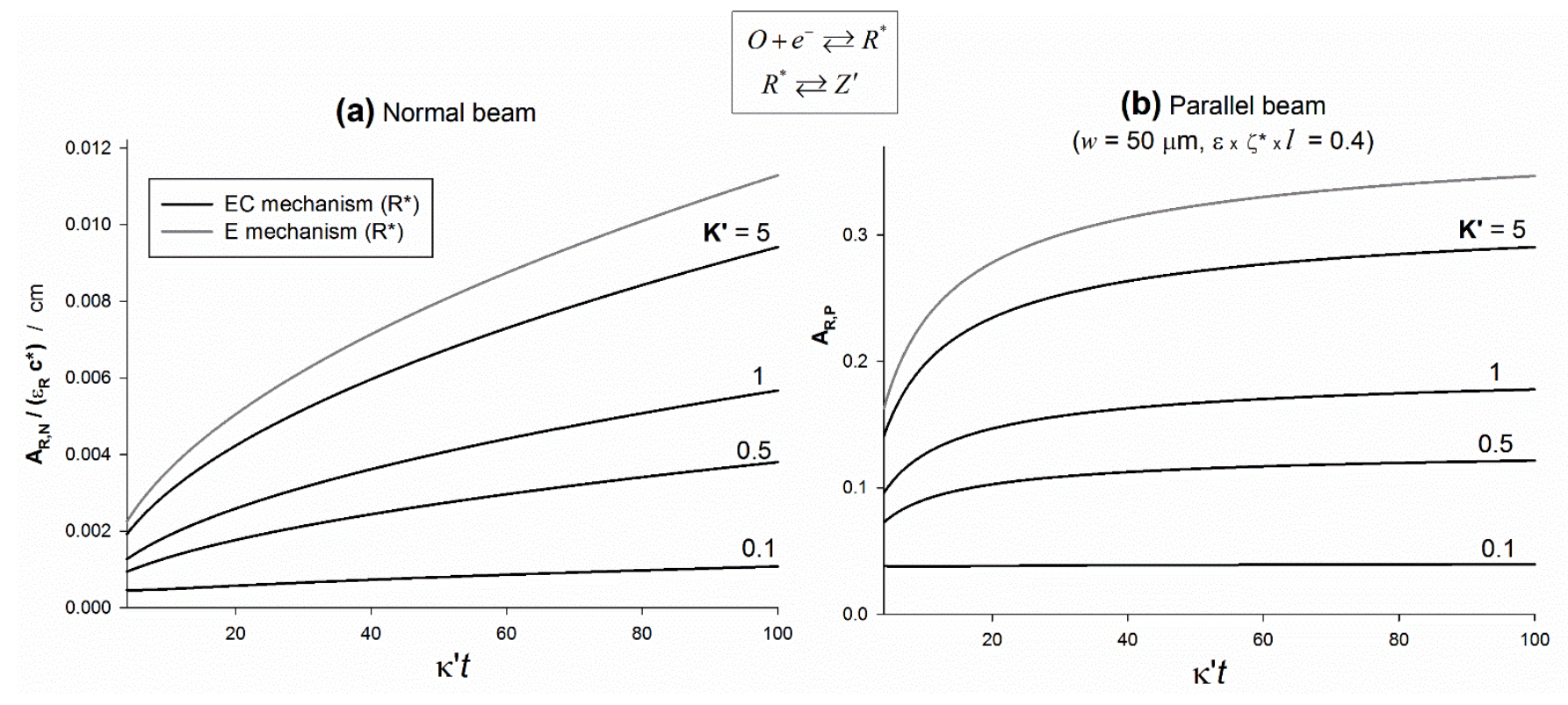

Figure 5. Dimensionless chronoamperometric SEC response of the EC mechanism with $\mathrm{R}$ being the absorbing species as a function of the chemical kinetics $\left(\kappa^{\prime}\right)$ and thermodynamics $\left(K^{\prime}\right)$ plotted using Eq. (74) for the normal beam configuration and Eqs. (2) and (56) for the parallel one. Other conditions as in Figure 1

\subsubsection{Ion transfer at ITIES}

$$
\begin{gathered}
\mathrm{X}^{+}(w) \underset{\mathrm{X}^{+*}}{\rightleftarrows}(o) \\
\mathrm{X}^{+^{*}}(o) \underset{+L}{\stackrel{\kappa^{\prime}, K^{\prime}}{\rightleftarrows}} \mathrm{XL}(o)
\end{gathered}
$$

In the study of the transfer of an ion $\mathrm{X}^{+}$across the interface between two immiscible electrolyte solutions (ITIES), it is frequent to use a very lipophilic ligand $L$ that facilitates the transfer (so-called facilitated ion transfer, scheme (76)). Also, the diffusivity of the ion in the water and organic media can be very different (i.e., $D \neq D^{\prime}$ ), especially when liquid membranes are employed. Both aspects are included in the theory here developed such that a facilitated 
transfer mechanism with different diffusion coefficients can be studied with the expressions given in Eqs. (73) and (74) with $\gamma \neq 1$.

In Figure 6, the influence of the diffusivity of the ion in the organic phase $\left(D^{\prime}\right)$ on the spectro-electrochemical response of the facilitated transfer of a cation $\mathrm{X}^{+}$is studied. As expected, Figure 6a shows how $\mathrm{X}^{+}$accumulates closer to the liquid|liquid interface $(s=0)$ as the value of $D^{\prime}$ decreases. While this does not affect the absorbance in the normal configuration (see inset in Figure 6), the SEC signal in the parallel configuration is quite sensitive and it shows a complex dependence with the shape of the concentration profile (see Eq. (2)).

$$
\begin{aligned}
& \mathrm{X}^{+}(w) \rightleftarrows \mathrm{X}^{+^{*}}(o) \quad-D / D^{\prime}=1 \\
& \mathrm{X}^{+*}(o) \stackrel{\mathrm{K}^{\prime} \cdot K^{\prime}}{\rightleftarrows} Z^{\prime}(o) ; K^{\prime}=1 \quad---\quad D / D^{\prime}=10 \\
& \kappa^{\prime}=10 s^{-1} \quad-----D / D^{\prime}=100
\end{aligned}
$$
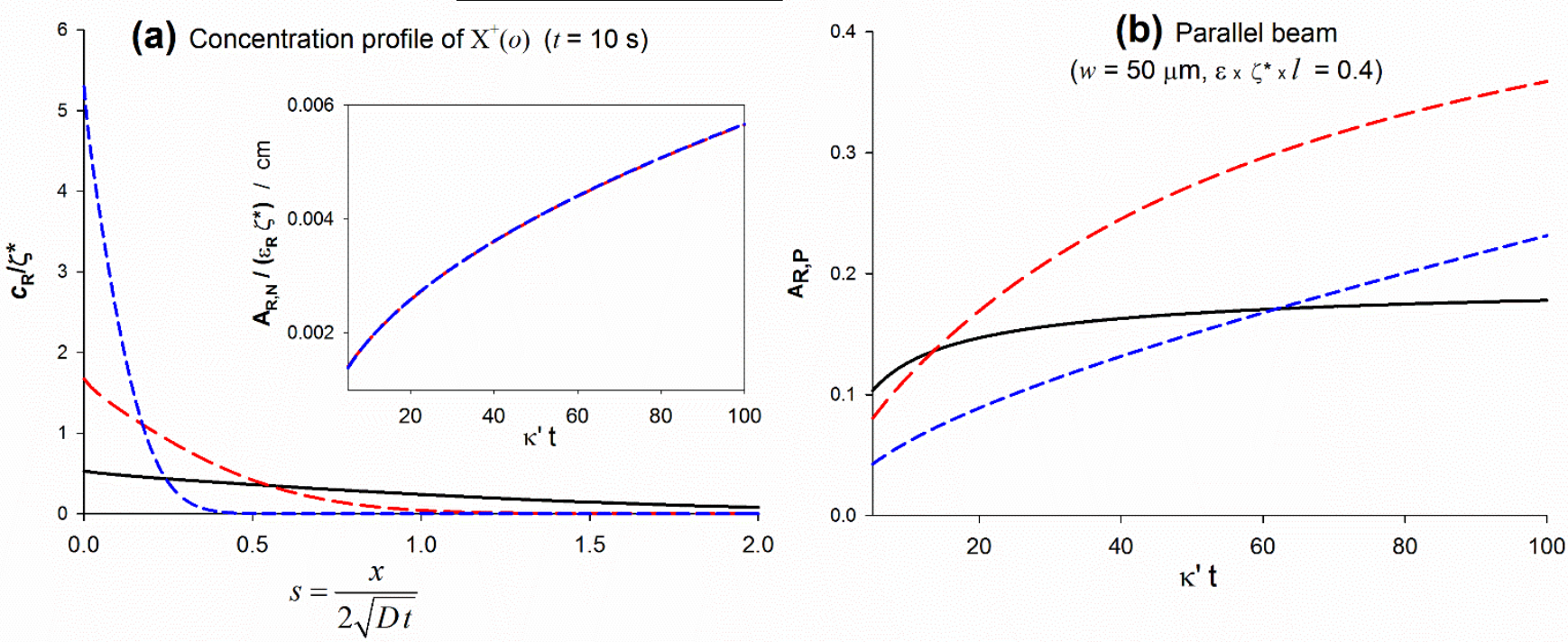

Figure 6. (a) Dimensionless concentration profile and normal-beam SEC response (inset) and (b) parallelbeam SEC response associated with the free cation $\mathrm{X}^{+}$in the organic phase for three different values of $D^{\prime}: D^{\prime}=D$ (black line); $D^{\prime}=0.1 D$ (red line) and $D^{\prime}=0.01 D$ (blue line). Eq. (74) for the normal beam configuration and Eqs. (2) and (56) for the parallel one and the concentration profiles. Limiting current conditions $\left(\mathrm{e}^{\eta} \rightarrow 0\right)$

The cases above-considered point out how spectrolectrochemistry can greatly assist in the elucidation and characterization of the kinetics (rate laws and rate constants), reaction mechanism (identification of intermediates and products of charge transfer process) and mass transport conditions of electrochemical systems. First, SEC adds a new source of information for the study of complex situations and for giving further consistency to the results of the 
electrochemical analysis. Specifically, the use of SEC is of particular value in those cases where the current response is not informative about some of the ongoing physicochemical processes, such as comproportionation/disproportionation reactions in multi-electron transfers or the chemical reactivity and diffusivity of the product of irreversible electrode processes. Finally, it is worth noting that SEC enables the determination of the absorbing properties of (highly) unstable species that are electrogenerated in situ. 


\section{Conclusions}

Analytical explicit expressions for the concentration profiles of the reaction mechanisms CEC, CE and EC have been deduced and applied to the analysis of the spectroelectrochemical (SEC) UV-vis signal. With the expressions here reported, it is possible to relate the absorbance transient of any of the species to the physicochemical dynamics of the system (specifically, the mass transport and homogeneous chemical kinetics) upon the application of constant potential pulse perturbation of any value. Two possibilities have been considered namely that the beam light can be incident either in a normal or in a parallel way to the electrode.

The equations presented provide accurate results for $\left(k_{1}+k_{2}\right) t>5$. For faster chemical reactions, the diffusive steady state treatment (dkss) has also been employed to obtain very simple closed-form expressions for the concentration profiles and for the absorbance transients. The influence of the chemical kinetics on the SEC signal under limiting current conditions ( $e^{\eta} \rightarrow 0$ ) has been analysed for the CE and EC mechanisms when only the electrogenerated species is photoactive. The latter case is particularly interesting since in such situations the electrochemical signal is not sensitive to the coupled chemical reaction. The value of the SEC parallel configuration, especially when the photoactive species is initially present in solution, has also been discussed. Finally, the study of ion transfer processes at ITIES via SEC has been considered pointing out that large differences between the ion diffusivity in each phase has an apparent effect on the parallel-beam signal in contrast to that in the normal configuration.

\section{Acknowledgments}

The authors greatly appreciate the financial support provided by the Fundación Séneca de la Región de Murcia (Project 19887/GERM/15) as well as by the Ministerio de Economía y Competitividad (Project CTQ-2015-65243-P). EL thanks the Ministerio de Economía y Competitividad for the fellowship "Juan de la Cierva-Incorporación 2015". JMGG thanks the Ministerio de Educación, Cultura y Deporte for the fellowship 'Ayuda de Formación de Profesorado Universitario 2015'. 


\section{Appendix A. Theoretical treatment}

We will make use of Kouteckýs dimensionless parameter method [24] to solve the boundary value problem corresponding to the CEC mechanism under linear, semi-infinite diffusion conditions (see Section 2). Thus, by introducing the dimensionless variables $s, s^{\prime}$ and $\chi$ (Eqs. (34)), the differential equation problem becomes into:

$$
\begin{aligned}
& \frac{\partial^{2} \varsigma}{\partial s^{2}}+2 s \frac{\partial \varsigma}{\partial s}-2 \chi \frac{\partial \varsigma}{\partial \chi}=0 \\
& \frac{\partial^{2} s^{\prime}}{\partial s^{\prime 2}}+2 s^{\prime} \frac{\partial s^{\prime}}{\partial s^{\prime}}-2 \chi \frac{\partial s^{\prime}}{\partial \chi}=0 \\
& \left.\begin{array}{l}
s \rightarrow \infty \\
s^{\prime} \rightarrow \infty
\end{array}\right\} \\
& \varsigma(\infty)=\varsigma^{*} \\
& \varsigma^{\prime}(\infty)=0 \\
& s=0 \\
& \left.s^{\prime}=0\right\}
\end{aligned}
$$

Following Koutecký's dimensionless parameters method [23,24], the solution for $\varsigma(s, \chi)$ is supposed to be a functional series of the variable $\chi$ as follows:

$$
\begin{aligned}
& \varsigma(s, \chi)=\sum_{j=0}^{\infty} \sigma_{j}(s) \chi^{j} \\
& \varsigma^{\prime}\left(s^{\prime}, \chi\right)=\sum_{j=0}^{\infty} \rho_{j}\left(s^{\prime}\right) \chi^{j}
\end{aligned}
$$

with:

$$
\begin{aligned}
& \sigma_{j}(s)=a_{j} \Psi_{j}(s)+\frac{\sigma_{j}(\infty) L_{j}(s)}{\lim _{s \rightarrow \infty} L_{j}(s)} \\
& \rho_{j}\left(s^{\prime}\right)=c_{j} \Psi_{j}\left(s^{\prime}\right)+\frac{\rho_{j}(\infty) L_{j}\left(s^{\prime}\right)}{\lim _{s^{\prime} \rightarrow \infty} L_{j}\left(s^{\prime}\right)}
\end{aligned}
$$

where functions $\Psi_{j}$ are given by: 


$$
\left.\Psi_{j}\left(s^{\left({ }^{\prime}\right)}\right)=\sum_{m=0}^{\infty} d_{j, m}\left(s^{\left({ }^{\prime}\right)}\right)^{m}-p_{j} \sum_{m=0}^{\infty} e_{j, m}\left(s^{(')}\right)^{m+1}\right\}
$$

having the following properties:

$$
\left.\begin{array}{l}
\Psi_{j}(0)=1 \\
\Psi_{j}(\infty)=0 \\
\Psi_{j}\left(s^{(\prime)}\right)=-p_{j} \Psi_{j-1}\left(s^{\left({ }^{\prime}\right)}\right) \\
\Psi_{0}\left(s^{(\prime)}\right)=\operatorname{erfc}\left(s^{(\prime)}\right)
\end{array}\right\}
$$

with coefficients $d_{j, m}$ and $e_{j, m}$ being given by Eqs. (38) and $p_{j}$ by Eq. (39). Also, $L_{j}$ are given by:

$$
\mathrm{L}_{\mathrm{j}}\left(s^{(\prime)}\right)=\sum_{\mathrm{m}=0}^{\infty} \mathrm{e}_{\mathrm{j}, \mathrm{m}}\left(s^{\left({ }^{\prime}\right)}\right)^{\mathrm{m}+1}
$$

Considering the application of the bulk conditions (Eq.(A2)) to Eqs. (A5)-(A6) leads to:

$$
\left.\begin{array}{l}
\sigma_{0}(\infty)=\varsigma^{*} \\
\sigma_{j \geq 1}(\infty)=0
\end{array}\right\}
$$

such that:

$$
\begin{aligned}
& \left\{\begin{array}{l}
\sigma_{0}(s)=a_{0} \Psi_{0}(s)+\varsigma^{*} \operatorname{erf}(s) \\
\sigma_{j}(s)=a_{j} \Psi_{j}(s) \quad(j>0)
\end{array}\right. \\
& \rho_{j}\left(s^{\prime}\right)=c_{j} \Psi_{j}\left(s^{\prime}\right) \quad \forall j
\end{aligned}
$$

Then, taking into account the surface conditions (see Eqs. (A3) and (A4)), Eqs. (A5) and (A11), the following expressions for coefficients $a_{j}$ are obtained:

$$
\begin{aligned}
& \left.\begin{array}{l}
\mathrm{c}_{0}=0 \\
\mathrm{a}_{0}=\varsigma^{*}
\end{array}\right\} \\
& \mathrm{c}_{\mathrm{j}}=-\mathrm{a}_{\mathrm{j}} \gamma \\
& \left.\mathrm{a}_{\mathrm{j} \geq 1}=\frac{\varsigma^{*}}{1+\mathrm{K}^{\prime} \mathrm{e}^{\eta} \gamma\left(\frac{1+\mathrm{K}}{1+\mathrm{K}^{\prime}}\right)} \frac{(-1)^{\mathrm{j}} 2^{\mathrm{j}}}{\prod_{1=1}^{\mathrm{j}} \mathrm{p}_{1}}\left(\frac{1+\mathrm{K}^{\prime} \mathrm{e}^{\eta} \gamma\left(\frac{1+\mathrm{K}}{1+\mathrm{K}^{\prime}}\right)}{\mathrm{K}+\sqrt{\frac{\kappa}{\kappa^{\prime}}} \mathrm{e}^{\eta} \gamma\left(\frac{1+\mathrm{K}}{1+\mathrm{K}^{\prime}}\right)}\right)\right\} \mathrm{j} \geq 1
\end{aligned}
$$

From Eqs. (A5), (A7)-(A8), (A11) and (A12), the concentration profiles of the oxidized species $\mathrm{O}$ and $Z$ can be calculated from Eqs.(43) and of the reduced species $R$ and $Z^{\prime}$ from Eqs. (44). 


\section{Appendix B. Major Symbols}

$\mathrm{A}(\lambda, t)$ UV-visible absorbance response.

$\mathrm{A}_{i, \mathrm{~N}}(\lambda, t)$ UV-visible absorbance response of species $i\left(i \equiv \mathrm{O}, \mathrm{Z}, \mathrm{R}\right.$ or $\left.\mathrm{R}^{\prime}\right)$ considering normal incidence of the light beam.

$\mathrm{A}_{i, N}^{d k s s}(\lambda, t)$ UV-visible absorbance response of species $i\left(i \equiv \mathrm{O}, \mathrm{Z}, \mathrm{R}\right.$ or $\left.\mathrm{R}^{\prime}\right)$, considering the normal incidence of the light beam for the CEC mechanism, obtained with the $d k s s$ treatment.

$\mathrm{A}_{i, \mathrm{P}}(\lambda, t) \quad$ UV-visible absorbance response of species $i\left(i \equiv \mathrm{O}, \mathrm{Z}, \mathrm{R}\right.$ or $\left.\mathrm{R}^{\prime}\right)$, considering incidence of the light beam parallel to the electrode surface.

$\mathrm{A}_{i, P}^{d k s s}(\lambda, t)$ UV-visible absorbance response of species $i\left(i \equiv \mathrm{O}, \mathrm{Z}, \mathrm{R}\right.$ or $\left.\mathrm{R}^{\prime}\right)$, considering the incidence of the light beam parallel to the electrode surface for the CEC mechanism, obtained with the $d k s s$ approach.

$c_{i}(x, t)$ Concentration profile of species $i\left(i \equiv \mathrm{O}, \mathrm{Z}, \mathrm{R}, \mathrm{R}^{\prime}\right)$.

$c_{i}^{*}$ Bulk concentration of species $i\left(i \equiv \mathrm{O}, \mathrm{Z}, \mathrm{R}, \mathrm{R}^{\prime}\right)$.

$D$ Diffusion coefficient.

$D^{\prime}$ Diffusion coefficient of the reduced species ( $R$ and $Z^{\prime}$ ).

$\varepsilon_{i}(\lambda)$ Wavelength dependent extinction coefficient of species $i\left(i \equiv \mathrm{O}, \mathrm{Z}, \mathrm{R}, \mathrm{R}{ }^{\prime}\right)$.

$\mathrm{K}$ (Conditional) chemical equilibrium constant of the reaction involving the oxidized species ( $\mathrm{O}$ and Z).

$\mathrm{K}^{\prime}$ (Conditional) chemical equilibrium constant of the reaction involving the reduced species ( $\mathrm{R}$ and $\left.Z^{\prime}\right)$.

$\kappa$ Sum of the chemical rate constants of the reaction involving the oxidized species.

$\kappa$ ' Sum of the chemical rate constants of the reaction involving the reduced species.

I Path length of the beam through the material sample in the normal mode. Path length of the beam over the electrode surface in the parallel configuration.

$\delta$ Thickness of the linear diffusion layer for the oxidized species ( $O$ and $Z$ ).

$\delta^{\prime}$ Thickness of the linear diffusion layer for the reduced species ( $R$ and $\left.Z^{\prime}\right)$.

$\delta_{r}$ Thickness of the linear reaction layer related to the perturbation of the chemical equilibrium involving the oxidized species ( $\mathrm{O}$ and $\mathrm{Z}$ ).

$\delta_{r}^{\prime}$ Thickness of the linear reaction layer related to the perturbation of the chemical equilibrium involving the reduced species ( $R$ and $\left.Z^{\prime}\right)$.

$w$ Height of the light beam in the parallel configuration.

$\varsigma^{*}$ Sum of the bulk concentration of the oxidized species ( $O$ and $Z$ ). 


\section{References}

[1] B.A. Coles, R.G. Compton, Photoelectrochemical ESR, J. Electroanal. Chem. Interfacial Electrochem. 144 (1983) 87-98.

[2] R.G. Compton, D.J. Page, G.R. Sealy, In-situ electrochemical ESR: First-order kinetics and transient signals, J. Electroanal. Chem. Interfacial Electrochem. 163 (1984) 65-75.

[3] W. Kaim, J. Fiedler, Spectroelectrochemistry: the best of two worlds, Chem. Soc. Rev. 38 (2009) 3373-3382.

[4] J.J. Walsh, R.J. Forster, T.E. Keyes, Spectroscopy of Electrochemical Systems, in: Springer Handb. Electrochem. Energy, Springer Berlin Heidelberg, Berlin, Heidelberg, 2017: pp. 365-421.

[5] L. Dunsch, Recent Advances in in situ multi-spectroelectrochemistry, J. Solid State Electrochem. 15 (2011) 1631-1646.

[6] D.A. Scherson, Y. V. Tolmachev, I.C. Stefan, D.A. Scherson, Y. V. Tolmachev, I.C. Stefan, Ultraviolet/Visible Spectroelectrochemistry, in: Encycl. Anal. Chem., John Wiley \& Sons, Ltd, Chichester, UK, 2006.

[7] A. Martínez, A. Colina, R.A.W. Dryfe, V. Ruiz, Spectroelectrochemistry at the liquid|liquid interface: Parallel beam UV-vis absorption, Electrochim. Acta. 54 (2009) 5071-5076.

[8] A.J. Bard, L.R. Faulkner, Electrochemical Methods: Fundamentals and Applications, 2nd ed., Wiley, New York, 2000.

[9] W. Wanzhi, X. Qingji, Y. Shouzhuo, Theory and application of analytical spectroelectrochemistry with long path length spectroelectrochemical cells, J. Electroanal. Chem. 328 (1992) 9-20.

[10] R. Kant, M.M. Islam, Theory of absorbance transients of an optically transparent rough electrode, J. Phys. Chem. C. 114 (2010) 19357-19364.

[11] J.F. Tyson, T.S. West, Analytical aspects of absorption spectroelectrochemistry at a platinum electrode-II Quantitative basis and study of organic compounds, Talanta 27 (1980) 335-342.

[12] N. Winograd, H.N. Blount, T. Kuwana, Spectroelectrochemical measurement of chemical reaction rates. First-order catalytic processes, J. Phys. Chem. 73 (1969) 3456-3462.

[13] Y. Xie, S. Dong, Theory of analytical spectroelectrochemistry, J. Electroanal. Chem. Interfacial Electrochem. 291 (1990) 1-10.

[14] A. Colina, J. Lopez-Palacios, A. Heras, V. Ruiz, L. Fuente, Digital simulation model for bidimensional spectroelectrochemistry, J. Electroanal. Chem. 553 (2003) 87-95.

[15] S. Ma, Y. Wu, Z. Wang, Spectroelectrochemistry for a coupled chemical reaction in the channel cell. Part I. Theoretical simulation of an EC reaction, J. Electroanal. Chem. 464 (1999) 176-180.

[16] C. Amatore, L. Nadjo, J.M. Savéant, Convolution and finite difference approach, J. Electroanal. Chem. Interfacial Electrochem. 90 (1978) 321-331.

[17] M.K. Hanafey, R.L. Scott, T.H. Ridgway, C.N. Reilley, Analysis of electrochemical mechanisms by finite difference simulation and simplex fitting of double potential step current, charge, and absorbance responses, Anal. Chem. 50 (1978) 116-137. 
[18] K. Ogura, T. Naito, Absorbance-time relationship at optically transparent electrode, Electrochim. Acta. 27 (1982) 1243-1246.

[19] D.H. Evans, M.J. Kelly, Comment on "Absorbance-time relationship at optically transparent electrode," Electrochim. Acta. 28 (1983) 1167.

[20] A. Molina, E. Torralba, C. Serna, J.A. Ortuño, Analytical solution for the facilitated ion transfer at the interface between two immiscible electrolyte solutions via successive complexation reactions in any voltammetric technique: Application to square wave voltammetry and cyclic voltammetry, Electrochim. Acta. 106 (2013) 244-257.

[21] E.J.F. Dickinson, J.G. Limon-Petersen, N. V. Rees, R.G. Compton, How Much Supporting Electrolyte Is Required to Make a Cyclic Voltammetry Experiment Quantitatively "Diffusional"? A Theoretical and Experimental Investigation, J. Phys. Chem. C. 113 (2009) 11157-11171.

[22] C. Amatore, K. Knobloch, L. Thouin, Alteration of diffusional transport by migration and natural convection. Theoretical and direct experimental evidences upon monitoring steady-state concentration profiles at planar electrodes, J. Electroanal. Chem. 601 (2007) $17-28$.

[23] I. Morales, A. Molina, Analytical expressions of the I-E-t curves of a CE process with a fast chemical reaction at spherical electrodes and microelectrodes, Electrochem. Commun. 8 (2006) 1453-1460.

[24] J. Koutecký, J. Čížek, Anwendung der methode der dimensionslosen parameter für die lösung von transportproblemen bei der elektrolyse mit konstantem strom an flacher und kugelförmiger elektrode, Collect. Czechoslov. Chem. Commun. 22 (1957) 914-928.

[25] Á. Molina, J. González, Pulse Voltammetry in Physical Electrochemistry and Electroanalysis, Monographs, Springer International Publishing, Cham, 2016.

[26] Á. Molina, F. Martínez-Ortiz, E. Laborda, I. Morales, Rigorous analytical solution for a preceding chemical reaction in Normal Pulse Voltammetry at spherical electrodes and microelectrodes, J. Electroanal. Chem. 633 (2009) 7-14.

[27] Á. Molina, E. Torralba, C. Serna, F. Martínez-Ortíz, E. Laborda, Some insights into the facilitated ion transfer voltammetric responses at ITIES exhibiting interfacial and bulk membrane kinetic effects., Phys. Chem. Chem. Phys. 14 (2012) 15340-54.

[28] J. Garoz-Ruiz, A. Heras, A. Colina, Direct Determination of Ascorbic Acid in a Grapefruit: Paving the Way for In Vivo Spectroelectrochemistry, Anal. Chem. 89 (2017) 1815-1822.

[29] R.G. Compton, C.E. Banks, Understanding Voltammetry, 2nd ed., Imperial College Press, London, 2010. 